\title{
APPROXIMATION OF JENSEN MEASURES BY IMAGE MEASURES UNDER HOLOMORPHIC FUNCTIONS AND APPLICATIONS
}

\author{
SHANGQUAN BU AND WALTER SCHACHERMAYER
}

\begin{abstract}
We show that Jensen measures defined on $\mathbb{C}^{n}$ or more generally on a complex Banach space $X$ can be approximated by the image of Lebesgue measure on the torus under $X$-valued polynomials defined on $\mathbb{C}$. We give similar characterizations for Jensen measures in terms of analytic martingales and Hardy martingales. The results are applied to approximate plurisubharmonic martingales by Hardy martingales, which enables us to give a characterization of the analytic Radon-Nikodym property of Banach spaces in terms of convergence of plurisubharmonic martingales, thus solving a problem of G. A. Edgar.
\end{abstract}

\section{INTRODUCTION}

Let $X$ be a Banach space and $\mu$ a Radon probability measure on $X$ with first moment (i.e., $\left.\int_{X}\|x\| d \mu(x)<\infty\right)$. It is well known that there is a unique $x_{0} \in X$, called the barycenter of $\mu$ verifying

$$
f\left(x_{0}\right) \leq \int_{X} f(x) d \mu(x)
$$

for every real-valued convex Lipschitz function on $X$. We then call $\mu$ a Choquet measure for $x_{0}$.

Recall the following easy folklore result (compare [E3] for further results in this context; unexplained notation will be defined below):

Theorem (0). Let $\mu$ be a Radon probability measure with first moment on a Banach space $X$ and $x_{0} \in X$. The following are equivalent:

(i) $\mu$ is a Choquet measure with barycenter $x_{0}$.

(ii) There is a Bochner integrable function $f:[0,1] \rightarrow X$ with expectation $\mathbb{E}(f)=\int_{0}^{1} f(x) d \lambda(x)=x_{0}$ and such that the image measure $f(\lambda)$ equals $\mu$.

(iii) $\mu$ can be approximated in the narrow topology with respect to the class of Lipschitz functions on $X$ by the final distribution $D_{n}(\mathbb{P})$ of a finite dyadic martingale $\left(D_{i}\right)_{i=0}^{n}$ defined on a probability space $(\Omega, \Sigma, \mathbb{P})$ and starting at $x_{0}$, i.e., $D_{0} \equiv x_{0}$.

(iv) $\mu$ equals the final distribution $M_{n}(\mathbb{P})$ of a finite martingale $\left(M_{i}\right)_{i=0}^{n}$ defined on a probability space $(\Omega, \Sigma, \mathbb{P})$ and starting at $x_{0}$, i.e., $M_{0} \equiv x_{0}$.

Received by the editors January 11, 1989 and, in revised form, February 7, 1990.

1980 Mathematics Subject Classification (1985 Revision). Primary 46B20, 32F05, 60 G46.

Key words and phrases. Plurisubharmonic martingales, Hardy martingales, Jensen measures, the analytic Radon-Nikodym property. 
We have stated the rather easy Theorem $(0)$ as it is the "real" analogue of the "complex" Theorem (A) below, where the term Choquet measure is replaced by Jensen measure, integrable function by holomorphic function, dyadic martingale by analytic martingale and the term martingale in (iv) by Hardy martingale (unexplained notions will be defined in the subsequent section):

Theorem (A). Let $\mu$ be a Radon probability measure with first moment on a complex Banach space $X$ and $x_{0} \in X$. Equip $\mathscr{M}^{1}(X)$ with the weak topology induced by $\operatorname{Lip}(X)$. The following are equivalent:

(i) $\mu$ is a Jensen measure on $X$ with barycenter $x_{0}$.

(ii) $\mu$ is in the closure of the subset

$$
\mathbf{P}=\left\{g(\lambda): g: \mathbb{C} \rightarrow X \text { is a polynomial with } g(0)=x_{0}\right\}
$$

of $\mathscr{M}^{1}(X)$.

(iii) $\mu$ is in the closure of the subset

$$
\mathbf{A}=\left\{F_{n}\left(\lambda^{n}\right):\left(F_{i}\right)_{i=0}^{n} \text { is an analytic martingale with } F_{0} \equiv x_{0}\right\}
$$

of $\mathscr{M}^{1}(X)$.

(iv) $\mu$ is in the closure of the subset

$$
\mathbf{H}=\left\{F_{n}\left(\lambda^{n}\right):\left(F_{i}\right)_{i=0}^{n} \text { is a Hardy martingale with } F_{0} \equiv x_{0}\right\}
$$

of $\mathscr{M}^{1}(X)$.

Remark. The implication (ii) $\Rightarrow$ (i), (iii) $\Rightarrow$ (i) and (iv) $\Rightarrow$ (i) are easily seen to be true. The equivalence of (ii) and (iii) has essentially been proved by G. A. Edgar [E2] while the equivalence of (ii) and (iv) follows from a theorem of N. Ghoussoub and B. Maurey [G-M, Theorem 4.1].

The decisive new information given by Theorem (A) is how to approximate an abstract Jensen measure by analytic objects as in (ii), (iii) or (iv). Note that the only analytic concept appearing in the definition of Jensen measures is that of plurisubharmonic functions, against which $\mu$ is tested via inequality (1) below.

We are afraid that the reader interested in several complex variables might be turned off by the infinite-dimensional setting and the concepts of analytic and Hardy martingales. We therefore formulate another version of Theorem (A), which is more in the spirit of several complex variables and we give a proof which does not rely on the concepts of analytic and Hardy martingales. However, the basic idea of the proof is the same as in Theorem (A).

Theorem (B). Let $U$ be a domain in $\mathbb{C}^{n}$ and $\mu$ a probability measure with compact support in $U$. Then $\mu$ is a Jensen measure on $U$ with barycenter $x_{0} \in U$ if and only if $\mu$ can be approximated by image measure $g(\lambda)$, where $g$ is polynomials $g: \mathbb{C} \rightarrow \mathbb{C}^{n}, g(\overline{\mathbb{D}}) \subseteq U, g(0)=x_{0}$ in the following sense: For every finite set $\left\{f_{1}, f_{2}, \ldots, f_{m}\right\}$ of continuous functions on $U$ and $\varepsilon>0$ there is $g$ as above such that for $1 \leq i \leq m$,

$$
\left|\left\langle f_{i}, \mu\right\rangle-\left\langle f_{i}, g(\lambda)\right\rangle\right|=\left|\int_{U} f_{i}(x) d \mu(x)-\int_{0}^{1} f_{i} \circ g\left(e^{2 \pi i \theta}\right) d \theta\right|<\varepsilon .
$$

Let us come back again to the context of Banach spaces: it was proved by G. A. Edgar [E2] that convergence of $X$-valued $L^{1}$-bounded analytic martingales characterizes the analytic Radon-Nikodym property of $X$ introduced by 
A. Bukhvalov and A. Danilevich [Bu-Da] and the corresponding theorem for Hardy martingales has been observed by D. J. H. Garling [Gar, Theorem 3].

Note that the definition of analytic martingales as well as Hardy martingales refers to a special representation of these martingales, namely that they are defined on the probability space $\left(\mathbb{T}^{\mathbb{N}}, \lambda^{\mathbb{N}}\right)$. This is somehow unsatisfactory from a probabilistic point of view. G. A. Edgar has introduced the representation free concept of plurisubharmonic martingales (Definition I.8. below).

One easily verifies that a Hardy martingale (and therefore an analytic martingale) is a plurisubharmonic martingale [Gar, Theorem 1]. Theorem (C), which is the second main result of this paper, gives a kind of converse.

Theorem (C). Let $X$ be a complex Banach space, $\left(M_{n}\right)_{n=0}^{\infty}$ an $X$-valued plurisubharmonic martingale and $\left(\varepsilon_{n}\right)_{n=1}^{\infty}$ positive numbers. Then there is a representation $\left(F_{n}\right)_{n=0}^{\infty}$ of $\left(M_{n}\right)_{n=0}^{\infty}$ defined on $\left(\mathbb{T}^{\mathbb{N}}, \lambda^{\mathbb{N}}\right)$ such that $F_{n}$ depends only on the first $n$ coordinates of $\mathbb{T}^{\mathbb{N}}$ (and may therefore be identified with a function on $\left.\mathbb{T}^{n}\right)$ and a Hardy martingale $\left(G_{n}\right)_{n=0}^{\infty}$ such that, for every $n \in \mathbb{N}$,

$$
\left\|\left(F_{n}-F_{n-1}\right)-\left(G_{n}-G_{n-1}\right)\right\|_{L^{1}\left(\mathbb{T}^{n}, X\right)}<\varepsilon_{n} .
$$

The solution to the problem of G. A. Edgar [E2] now follows immediately from Theorem $(\mathrm{C})$. This result has also been proved by N. Ghoussoub and B. Maurey [G-M] by different methods.

Corollary (D). A Banach space $X$ has the analytic Radon-Nikodym property if and only if $L^{1}$-bounded $X$-valued plurisubharmonic martingales converge almost surely.

We now describe the organization of this paper.

In $\S 1$ we gather the necessary definitions and notations. In $\S 2$ we prove Theorem (A). The proof turns out to be surprisingly simple and uses the HahnBanach theorem in a crucial way.

In $\S 3$ we prove Theorem (B), which is formulated in the local setting (i.e., for domains in $\mathbb{C}^{n}$ ) and we therefore also prove some technical results.

In $\S 4$ we prepare the tools needed for Theorem $(\mathrm{C})$ : A more precise and parametrised version of Theorem (A) is proved (Proposition IV.2) and we have to use some techniques from measure theory (disintegration of measures, measurable selections).

In $\S 5$ we then prove Theorem (C) and Corollary (D). We also note an application of Theorem (C) to Analytic Martingale Transform spaces introduced by D. J. H. Garling [Gar], extending a result of $\mathrm{Xu}$ [X].

For unexplained notation we refer to [L-T] for the Banach space concepts and to $[\mathrm{Ra}]$ or $[\mathrm{K}]$ for the concepts of several complex variables.

Acknowledgement. We thank B. Maurey for substantial help in establishing a proof of Corollary (D) and we also thank N. Ghoussoub, P. Müller and E. Perkins for their advice in the preparation of this paper.

\section{Definitions AND NOTATIONS}

Throughout this paper, $\mathbb{T}$ will denote the torus $\left\{e^{2 \pi i \theta}: 0 \leq \theta<1\right\}$ which we shall freely identify with a subset of $\mathbb{C}$ or with $[0,1[$. The open (resp. closed) disc of $\mathbb{C}$ will be denoted by $\mathbb{D}$ (resp. $\overline{\mathbb{D}}$ ). Normalized Lebesgue measure on $\mathbb{T}$ as well as on $[0,1]$ will always be denoted by $\lambda$. 
$X$ will denote a complex Banach space; we shall consider Radon probability measures on $X$ (see e.g., [Schw2]). As every Radon probability measure $\mu$ is supported by a separable subspace of $X$ we shall assume throughout the paper without loss of generality that $X$ is separable, hence the set of Radon probability measures on $X$ coincides with the set of all probability measures defined on the Borel $\sigma$-field generated by the metric topology of $X$ [Schw2]. If $\mu$ is a measure with first moment on $X$ (i.e, $\left.\int\|x\| d \mu(x)<\infty\right)$ and $\phi: X \rightarrow \mathbb{R}$ is a Lipschitz function, then we may form

$$
\langle\phi, \mu\rangle=\int_{X} \phi(x) d \mu(x) .
$$

If $U$ is a domain in $\mathbb{C}^{n}, \mu$ is a Radon measure on $U$ and $\phi: U \rightarrow \mathbb{R}$ is a measurable function, we shall also denote the scalar product as above if the right term makes sense.

If $U$ is a domain in $\mathbb{C}$, a function $g: U \rightarrow X$ is called analytic (or holomorphic) if, for every $x^{*} \in X^{*}, x^{*} \circ g$ is analytic. A function $g: \mathbb{C} \rightarrow X$ of the form $g(z)=\sum_{n=0}^{N} x_{n} z^{n}$ with $x_{n} \in X$ and $N \in \mathbb{N}$ will be called an $X$-valued polynomial on $\mathbb{C}$. Note that, if $U$ contains $\overline{\mathbb{D}}$ and $g: U \rightarrow X$ is analytic then we can approximate $g$ by $X$-valued polynomials uniformly on $\overline{\mathbb{D}}$ (see e.g., [Ch]).

We shall denote for $1 \leq p \leq \infty$ by $L^{p}(\mathbb{T}, X)$ the space of Bochner integrable functions $f: \mathbb{T} \rightarrow X$ equipped with the norm

$$
\|f\|_{p}=\left(\int_{0}^{1}\left\|f\left(e^{2 \pi i \theta}\right)\right\|^{p} d \theta\right)^{1 / p}
$$

for $1 \leq p<\infty$ and for $p=\infty$

$$
\|f\|_{\infty}=\underset{t \in \mathbb{T}}{\operatorname{ess} \sup }\|f(t)\|,
$$

and by $H_{0}^{p}(\mathbb{T}, X)$ the subspace of $L^{p}(\mathbb{T}, X)$ formed by the elements $g$ verifying, for every $k \geq 0$,

$$
\int_{0}^{1} e^{2 k \pi i \theta} f\left(e^{2 \pi i \theta}\right) d \theta=0 .
$$

We shall identify elements $f \in H_{0}^{p}(\mathbb{T}, X)$ with functions on $\overline{\mathbb{D}}$, i.e., the analytic extension of $f$ to $\overline{\mathbb{D}}$ obtained via the Poisson kernel.

Denote by $\mathscr{M}^{1}(X)$ the space of finite measures on $X$ with first moment, i.e., $\int_{X}\|x\| d|\mu|(x)<\infty$, and by $\operatorname{Lip}(X)$ the space of Lipschitz functions on $X$. The scalar product $\langle$,$\rangle defined above places these spaces in duality and$ we shall equip $\mathscr{M}^{1}(X)$ with the weak topology induced by $\operatorname{Lip}(X)$.

If $(\Omega, \Sigma, \mathbb{P})$ is a probability space, $\left(\Omega^{\prime}, \Sigma^{\prime}\right)$ a measure space and $F: \Omega \rightarrow$ $\Omega^{\prime}$ a measurable map, we denote by $F(\mathbb{P})$ the image measure of $\mathbb{P}$ under $F$ which is defined, for $A \in \Sigma^{\prime}$, by

$$
F(\mathbb{P})(A)=\mathbb{P}\left(F^{-1}(A)\right) .
$$

Definition I.1. If $\left(\mu_{\alpha}\right)_{\alpha \in I}$ is a net of probability measures on a polish space $(E, d)$, we shall say that $\left(\mu_{\alpha}\right)_{\alpha \in I}$ converges narrowly or in the narrow topology 
to a probability measure $\mu$ if, for every bounded continuous function $f: E \rightarrow$ $\mathbb{R}$,

$$
\lim _{\alpha}\left\langle f, \mu_{\alpha}\right\rangle=\langle f, \mu\rangle .
$$

Definition I.2 [E1]. Let $U$ be a domain in $X$. A function $\phi: U \rightarrow \mathbb{R} \cup\{-\infty\}$ is called plurisubharmonic on $U$ if $\phi$ is upper semicontinuous and if for every $x, y \in X$ such that $\{x+\overline{\mathbb{D}} y\} \subseteq U$

$$
\phi(x) \leq \int_{0}^{1} \phi\left(x+e^{2 \pi i \theta} y\right) d \theta .
$$

Definition I.3 (compare [E1]). Let $X$ be a complex Banach space and $\mu$ a probability measure on $X$ with first moment. We say that $\mu$ is a Jensen measure on $X$ with barycenter $x_{0} \in X$ if, for every Lipschitz plurisubharmonic function, $\phi$ on $X$

$$
\phi\left(x_{0}\right) \leq \int_{X} \phi(y) d \mu(y) .
$$

Remark I.4. First note that there are more plurisubharmonic functions than convex Lipschitz functions on $X$ and therefore fewer Jensen measures than Choquet measures on $X$. For a general account on Jensen measures we refer to [Gam].

Classically Jensen measures are supposed to have compact support. In view of the application to $L^{1}$-bounded martingales (Theorem (C) below) we place ourselves into the more general context of measures with first moment; hence we have to restrict ourselves to require inequality (1) only for Lipschitz plurisubharmonic functions and not arbitrary plurisubharmonic functions to avoid integrability problems. However in the context of measures with compact support on a domain $U$ of $\mathbb{C}^{n}$ it will be more natural to adopt the subsequent concept:

Definition I.5. Let $U$ be a domain in $\mathbb{C}^{n}$ and $\mu$ a probability measure with compact support $K$ in $U$. We say that $\mu$ is a Jensen measure on $U$ with barycenter $x_{0} \in U$ if, for every plurisubharmonic function $\varphi: U \rightarrow \mathbb{R} \cup\{-\infty\}$ we have

$$
\varphi\left(x_{0}\right) \leq \int_{U} \varphi(x) d \mu(x) .
$$

Remark I.6. Note that by the upper semicontinuity of $\varphi$ the integral on the right-hand side is well defined (with values in $\mathbb{R} \cup\{-\infty\}$ ). The definition is more in the classical spirit of Jensen measures and does not refer to Lipschitz functions as Definition I.3 above. We shall show in Proposition III.4 below that these two definitions are consistent.

Definition I.7. For a Banach space $X$, a sequence of functions $\left(F_{n}\right)_{n=0}^{\infty}, F_{0} \equiv$ $x_{0}, F_{n} \in L^{1}\left(\mathbb{T}^{n}, \lambda^{n}, X\right)$ (where $\mathbb{T}^{0}$ is a one point space) is called an $X$-valued

(a) analytic martingale ([Bo-Da], see also [D-G-T] and [E1]) if, for $n \in \mathbb{N}$ and $\left(\theta_{1}, \theta_{2}, \ldots, \theta_{n-1}\right) \in \mathbb{T}^{n-1}$

$$
F_{n}\left(\theta_{1}, \theta_{2}, \ldots, \theta_{n}\right)-F_{n-1}\left(\theta_{1}, \theta_{2}, \ldots, \theta_{n-1}\right)=f_{n}\left(\theta_{1}, \theta_{2}, \ldots, \theta_{n-1}\right) e^{2 \pi i \theta_{n}} ;
$$


(b) Hardy martingale ([Gar], see also [G-M]) if, for every $n \in \mathbb{N}$ and $\left(\theta_{1}, \theta_{2}\right.$, $\left.\ldots, \theta_{n-1}\right) \in \mathbb{T}^{n-1}$, the function

$$
\theta_{n} \rightarrow d_{n}\left(\theta_{1}, \theta_{2}, \ldots, \theta_{n-1}, \theta_{n}\right),
$$

defined by

$$
\begin{aligned}
& d_{n}\left(\theta_{1}, \theta_{2}, \ldots, \theta_{n-1}, \theta_{n}\right) \\
& \quad=F_{n}\left(\theta_{1}, \theta_{2}, \ldots, \theta_{n-1}, \theta_{n}\right)-F_{n-1}\left(\theta_{1}, \theta_{2}, \ldots, \theta_{n-1}\right),
\end{aligned}
$$

is in $H_{0}^{1}(\mathbb{T}, X)$.

If $U$ is a domain in $X$ we call $\left(F_{n}\right)_{n=1}^{\infty}$ a $U$-valued analytic (resp. Hardy) martingale if in addition to the above requirements, for every $n \in \mathbb{N}$ and $\left(\theta_{1}, \theta_{2}, \ldots, \theta_{n-1}\right) \in \mathbb{T}^{n-1}$,

$$
\begin{aligned}
& F_{n-1}\left(\theta_{1}, \theta_{2}, \ldots, \theta_{n-1}\right)+f_{n}\left(\theta_{1}, \theta_{2}, \ldots, \theta_{n-1}\right) r e^{2 \pi i \theta_{n}} \in U, \\
& \quad\left(\text { resp. } F_{n-1}\left(\theta_{1}, \theta_{2}, \ldots, \theta_{n-1}\right)+d_{n}\left(\theta_{1}, \theta_{2}, \ldots, \theta_{n-1}, r e^{2 \pi i \theta_{n}}\right) \in U\right),
\end{aligned}
$$

for every $0 \leq r \leq 1$ and $\theta_{n} \in \mathbb{T}$.

Obviously analytic martingales are Hardy martingales. The term martingale is justified as one may identify $\left(F_{n}\right)_{n=0}^{\infty}$ in an obvious way with a stochastic process on $\left(\mathbb{T}^{\mathbb{N}}, \lambda^{\mathbb{N}}\right)$ equipped with its natural filtration $\left(\Sigma_{n}\right)_{n=0}^{\infty}$ which is readily verified to be a martingale (see [Gar]).

Definition I.8 (compare [E1]). An $X$-valued martingale $\left(M_{n}\right)_{n=0}^{\infty}$, defined on a probability space $(\Omega, \Sigma, \mathbb{P})$ and such that $M_{0} \equiv x_{0}$ is called a plurisubharmonic martingale if, for every Lipschitz plurisubharmonic function $\phi$ on $X$, the stochastic process $\left(\phi \circ M_{n}\right)_{n=0}^{\infty}$ is a submartingale.

One can easily observe that Hardy martingales (whence, in particular, analytic martingales) are plurisubharmonic martingales.

To end this section let us point out the easy implications among the above concepts: Let $g \in H_{0}^{1}(\mathbb{T}, X)$ and identify $g$ with a function $g$ on $\overline{\mathbb{D}}$ which is analytic in $\mathbb{D}$. Then the image measure $g(\lambda)$ is a Jensen measure on $X$ with barycenter 0 . Indeed, if $\phi: X \rightarrow \mathbb{R}$ is a Lipschitz plurisubharmonic function then $\phi \circ g$ is subharmonic on $\mathbb{D}$ and the radial limits exist almost surely; therefore

$$
\phi(0)=\phi \circ g(0) \leq \int_{0}^{1} \phi \circ g\left(e^{2 \pi i \theta}\right) d \theta=\int_{X} \phi(x) d(g(\lambda))(x) .
$$

Similarly one verifies that for a finite Hardy martingale on $X$ (whence, in particular, for a finite analytic martingale) $\left(F_{i}\right)_{i=0}^{n}$, with $F_{0} \equiv x_{0}$, the image measure $F_{n}\left(\lambda^{n}\right)$ is a Jensen measure on $X$ with barycenter $x_{0}$ (compare [Gar, Theorem 1]).

This remark takes care of the easy implications of Theorem (A) above. In the next section we shall prove that the reverse implications also hold true.

\section{THE PROOF OF THEOREM (A)}

Recall the characterization of the plurisubharmonic hull of a function which has been proved by G. A. Edgar [E1, Lemma 2.1]. We give a version formulated for domains (compare also Proposition III.4 below) and use a slightly weaker hypothesis than in [E1]: 
Proposition II.1. Let $U \subseteq X$ be a domain and $f: U \rightarrow \mathbb{R} \cup\{-\infty\}$ an upper semicontinuous function. Define $f_{0}=f$ and for $n \geq 1$

$$
f_{n}(x)=\inf \left\{\int_{0}^{1} f_{n-1}\left(x+e^{2 \pi i \theta} y\right) d \theta\right\}
$$

there the inf is taken over all $y \in X$ such that $\{x+\overline{\mathbb{D}} y\} \subseteq U$. Then $\left(f_{n}\right)_{n=0}^{\infty}$ decreases pointwise to the largest plurisubharmonic function $\hat{f}$ on $U$ dominated by $f$.

Proof. It is obvious that $\left(f_{n}\right)_{n=0}^{\infty}$ decreases. We verify inductively that $f_{n}$ is upper semicontinuous: $f_{0}=f$ is upper semicontinuous. Suppose $f_{n-1}$ is upper semicontinuous and let $\left(x_{k}\right)_{k=0}^{\infty}$ in $U$ be such that $\lim _{k \rightarrow \infty} x_{k}=x_{0}$. If $y_{0} \in X$ is such that $\left\{x_{0}+\overline{\mathbb{D}} y_{0}\right\} \subseteq U$ then there is $k_{0}$ such that $\left\{x_{k}+\overline{\mathbb{D}} y_{0}\right\} \subseteq U$ for $k \geq k_{0}$. The upper semicontinuous function $f_{n-1}$ is bounded above on the relatively compact set $\bigcup_{k=k_{0}}^{\infty}\left\{x_{k}+\overline{\mathbb{D}} y_{0}\right\}$ and, for every $z \in \overline{\mathbb{D}}$,

$$
f_{n-1}\left(x_{0}+z y_{0}\right) \geq \limsup _{k \rightarrow \infty} f_{n-1}\left(x_{k}+z y_{0}\right) .
$$

Hence we obtain from Fatou's lemma that, for every $y_{0} \in X$ verifying $\left\{x_{0}+\right.$ $\left.\overline{\mathbb{D}} y_{0}\right\} \subseteq U$,

$$
\begin{aligned}
\int_{0}^{1} f_{n-1}\left(x_{0}+e^{2 \pi i \theta} y_{0}\right) d \theta & \geq \limsup _{k \rightarrow \infty} \int_{0}^{1} f_{n-1}\left(x_{k}+e^{2 \pi i \theta} y_{0}\right) d \theta \\
& \geq \limsup _{k \rightarrow \infty} f_{n}\left(x_{k}\right),
\end{aligned}
$$

and therefore

$$
f_{n}\left(x_{0}\right) \geq \limsup _{k \rightarrow \infty} f_{n}\left(x_{k}\right) .
$$

This shows that each $f_{n}$ and therefore $\hat{f}$ is upper semicontinuous.

For every plurisubharmonic function $\phi$ on $U, \phi \leq f$, we have $\phi \leq f_{n}$ for every $n \in \mathbb{N}$. Indeed, clearly $\phi \leq f_{0}$ and suppose that $\phi \leq f_{n-1}$. Then for every $x_{0} \in U$ and $y_{0} \in X$ such that $\left\{x_{0}+\overline{\mathbb{D}} y_{0}\right\} \subseteq U$

$$
\int_{0}^{1} f_{n-1}\left(x_{0}+e^{2 \pi i \theta} y_{0}\right) d \theta \geq \int_{0}^{1} \phi\left(x_{0}+e^{2 \pi i \theta} y_{0}\right) \geq \phi\left(x_{0}\right),
$$

whence $f_{n}\left(x_{0}\right) \geq \phi\left(x_{0}\right)$, which gives the inductive step. Hence we conclude that $\hat{f} \geq \phi$ for every plurisubharmonic function $\phi$ on $U$ dominated by $f$.

Finally we have to show the mean value inequality for $\hat{f}$ which follows from the Beppo Levi's monotone convergence theorem: for $x_{0} \in U, y_{0} \in X$, with $\left\{x_{0}+\overline{\mathbb{D}} y_{0}\right\} \subseteq U$

$$
\begin{aligned}
\hat{f}\left(x_{0}\right) & =\lim _{n \rightarrow \infty} f_{n}\left(x_{0}\right) \leq \lim _{n \rightarrow \infty} \int_{0}^{1} f_{n-1}\left(x_{0}+e^{2 \pi i \theta} y_{0}\right) d \theta \\
& =\int_{0}^{1} \hat{f}\left(x_{0}+e^{2 \pi i \theta} y_{0}\right) d \theta .
\end{aligned}
$$

The proof is complete. 
Remark II.2. As noted by G. A. Edgar [E1] one also may write the definition of $f_{n}$ in the following way:

$$
\begin{aligned}
f_{n}(x)=\inf \left\{\mathbb{E}\left(f\left(F_{n}\right)\right):\left(F_{i}\right)_{i=0}^{n}\right. & \text { is a } U \text {-valued analytic } \\
& \text { martingale with } \left.F_{0} \equiv x\right\} .
\end{aligned}
$$

Proof of Theorem (A).

(i) $\Rightarrow$ (iii) We first show that the set

$$
\begin{aligned}
\mathbf{A}=\left\{F_{n}\left(\lambda^{n}\right):\left(F_{i}\right)_{i=0}^{n}\right. & \text { is an } X \text {-valued } \\
& \text { analytic martingale with } \left.F_{0} \equiv x_{0}\right\},
\end{aligned}
$$

is a convex subset of $\mathscr{M}^{1}(X)$ : let $\left(F_{i}^{\prime}\right)_{i=0}^{n}$ and $\left(F_{i}^{\prime \prime}\right)_{i=0}^{m}$ be two analytic martingales as above. We may assume $n=m$. Define now an analytic martingale $\left(F_{i}\right)_{i=0}^{n+1}$ by letting $F_{0} \equiv F_{1} \equiv x_{0}$ and for $1 \leq i \leq n$,

$$
F_{i+1}\left(\theta_{1}, \theta_{2}, \ldots, \theta_{i+1}\right)= \begin{cases}F_{i}^{\prime \prime}\left(\theta_{2}, \ldots, \theta_{i+1}\right) & \text { if } 0 \leq \theta_{1}<\frac{1}{2}, \\ F_{i}^{\prime}\left(\theta_{2}, \ldots, \theta_{i+1}\right) & \text { if } \frac{1}{2} \leq \theta_{1}<1 .\end{cases}
$$

Clearly

$$
F_{n+1}\left(\lambda^{n+1}\right)=\left\{F_{n}^{\prime}\left(\lambda^{n}\right)+F_{n}^{\prime \prime}\left(\lambda^{n}\right)\right\} / 2,
$$

thus showing the convexity of $\mathbf{A}$.

If the conclusion of (iii) were false then, by the Hahn-Banach theorem we could find a Lipschitz function $f$ on $X$ and reals $\alpha<\beta$ such that

$$
\langle f, \mu\rangle=\int_{X} f d \mu \leq \alpha,
$$

while

$$
\langle f, \nu\rangle=\int_{X} f d \nu \geq \beta,
$$

for every $\nu \in A$. Note that the last line can be rewritten as

$$
\int_{\mathbb{T}^{n}} f \circ F_{n} d \lambda^{n} \geq \beta
$$

for every $X$-valued analytic martingale starting at $x_{0}$, hence by the preceding remark

$$
\hat{f}\left(x_{0}\right) \geq \beta \text {. }
$$

This gives the desired contradiction as we obtain the absurd inequality

$$
\alpha \geq\langle f, \mu\rangle \geq\langle\hat{f}, \mu\rangle \geq \hat{f}\left(x_{0}\right) \geq \beta .
$$

Note that $\hat{f}$ is Lipschitz by a remark of N. Ghoussoub and B. Maurey (see [G-M]), so no integrability problems arise.

(iii) $\Rightarrow$ (iv) Obvious.

(iv) $\Rightarrow$ (ii) Let $\left(F_{i}\right)_{i=0}^{n}$ be an $X$-valued Hardy martingale, $F_{0} \equiv x_{0}$. By [G-M, Theorem 6.1] we may find for $\varepsilon>0$, a function $g^{\varepsilon}: V_{\varepsilon} \rightarrow X$, which is analytic in a neighbourhood $V_{\varepsilon}$ of $\overline{\mathbb{D}}, g^{\varepsilon}(0)=x_{0}$ and a continuous surjection $\pi: \mathbb{T} \rightarrow \mathbb{T}^{n}$ such that $\pi(\lambda)=\lambda^{n}$ and

$$
\int_{0}^{1}\left\|F_{n}\left(\pi\left(e^{2 \pi i \theta}\right)\right)-g^{\varepsilon}\left(e^{2 \pi i \theta}\right)\right\| d \theta<\varepsilon / 2 .
$$


Find an $X$-valued polynomial $p^{\varepsilon}$ such that, for $|z| \leq 1$,

$$
\left\|p^{\varepsilon}(z)-g^{\varepsilon}(z)\right\|<\varepsilon / 2 \text {. }
$$

If $f: X \rightarrow \mathbb{R}$ is a Lipschitz function with Lipschitz constant $M$, then

$$
\begin{aligned}
\left|\left\langle f, F_{n}\left(\lambda^{n}\right)\right\rangle-\left\langle f, p^{\varepsilon}(\lambda)\right\rangle\right| & =\left|\int_{0}^{1}\left(f \circ F_{n}\left(\pi\left(e^{2 \pi i \theta}\right)\right)-f \circ p^{\varepsilon}\left(e^{2 \pi i \theta}\right)\right) d \theta\right| \\
& \leq M \int_{0}^{1}\left\|F_{n}\left(\pi\left(e^{2 \pi i \theta}\right)\right)-p^{\varepsilon}\left(e^{2 \pi i \theta}\right)\right\| d \theta<M \varepsilon,
\end{aligned}
$$

which readily shows that $F_{n}\left(\lambda^{n}\right)$ is in the closure of the subset $\mathbf{P}$ in (ii).

(ii) $\Rightarrow$ (i) This implication follows from the argument in the first section and the observation that, for a net $\left(\mu_{\alpha}\right)_{\alpha \in I}$ of Jensen measures on $X$ with barycenter $x_{0}$ converging in $\mathscr{M}^{1}(X)$, the limit $\mu$ again is a Jensen measure on $X$ with barycenter $x_{0}$.

\section{THE PROOF OF THEOREM (B)}

We now turn to the setting of Theorem (B) for which we shall give a selfcontained proof. We start with an easy but crucial example.

Example III.1. Consider $X=\mathbb{C}$ and denote by $\lambda$ the Lebesgue measure on the torus $\mathbb{T} \subseteq \mathbb{C}$ and by $\delta_{0}$ the Dirac measure at 0 . The measure $\mu=\left(\lambda+\delta_{0}\right) / 2$ clearly is a Jensen measure on $\mathbb{C}$ with barycenter 0 .

However there is no polynomial $p: \mathbb{C} \rightarrow \mathbb{C}$ such that $p(\lambda)=\mu$. Indeed such a polynomial would have to equal zero on a subset of $\mathbb{T}$ of measure $1 / 2$ and therefore have to be identically zero, a contradiction. In fact, it is well known that any not identically zero function $f$ in $H^{\infty}(\mathbb{T})$ equals zero at most on a subset of $\mathbb{T}$ of measure 0 (compare the proof of Lemma III.2 below), hence $\mu$ is not the image measure $f(\lambda)$ of any $f$ in $H^{\infty}(\mathbb{T})$ either.

This shows that the set $\mathbf{P}$ appearing in Theorem (A) fails to be convex (contrary to the set $\mathbf{A}$ ). Note however that it follows from Theorem (A) that the closure of $\mathbf{P}$ equals the set of Jensen measures on $X$ and therefore is convex.

We shall need the following result related to outer functions:

Lemma III.2. Let $A \subseteq \mathbb{T}$ be compact with measure $\lambda(A)=\alpha, G$ be an open neighbourhood of $A$ in $\overline{\mathbb{D}}$, and $\varepsilon>0$. There is a sequence $\left(p_{n}\right)_{n=1}^{\infty}$ of $\mathbb{C}$-valued polynomials on $\mathbb{C}$, mapping $\mathbb{D}$ into $\mathbb{D}$ such that

(i) $p_{n}(0)=0$ for every $n \in \mathbb{N}$.

(ii) $\left|p_{n}(z)\right|<\varepsilon$ for $z \in \overline{\mathbb{D}} \backslash G$ and $n \in \mathbb{N}$.

(iii) $\left(p_{n}\left(\lambda 1_{A}\right)\right)_{n=1}^{\infty}$ converges narrowly to $\alpha \lambda$.

(iv) $\left(p_{n}\left(\lambda 1_{\mathbb{T} \backslash A}\right)\right)_{n=1}^{\infty}$ converges narrowly to $(1-\alpha) \delta_{0}$.

In particular $\left(p_{n}(\lambda)\right)_{n=1}^{\infty}$ converges narrowly to $\alpha \lambda+(1-\alpha) \delta_{0}$.

Proof. We may suppose $0<\alpha<1$. Fix $n \in \mathbb{N}$ and define $h_{n}$ on $\mathbb{T}$ by

$$
h_{n}\left(e^{2 \pi i \theta}\right)= \begin{cases}0 & \text { if } e^{2 \pi i \theta} \in A, \\ -n & \text { if } e^{2 \pi i \theta} \in \mathbb{T} \backslash A,\end{cases}
$$

and extend $h_{n}$ via the Poisson kernel to a function on $\overline{\mathbb{D}}$ which is harmonic on $\mathbb{D}$. Let $\hat{h}_{n}$ be its harmonic conjugate normalized by $\hat{h}_{n}(0)=0$ and let

$$
g_{n}=\exp \left(h_{n}+i \hat{h}_{n}\right)
$$


which is a function in $H^{\infty}(\mathbb{T})$. We have

$$
g_{n}(0)=\exp \left(h_{n}(0)\right)=e^{-\alpha n},
$$

and

$$
\begin{gathered}
e^{-n}<\left|g_{n}(z)\right|<1 \quad \text { for } z \in \mathbb{D}, \\
\left|g_{n}(z)\right|=1 \quad \text { for } z \in A, \\
\left|g_{n}(z)\right|=e^{-n} \quad \text { for } z \in \mathbb{T} \backslash A .
\end{gathered}
$$

The last two lines imply that any cluster point of the image measures $\left(g_{n}(\lambda)\right)_{n=1}^{\infty}$ in the narrow topology is necessarily of the form $\alpha \nu+(1-\alpha) \delta_{0}$ where $\nu$ is a measure supported by the torus $\mathbb{T}$. We shall show that $\nu$ necessarily equals Lebesgue measure. We thank B. Maurey for providing the following proof which is simpler than the original one.

As $g_{n}$ is analytic on $\mathbb{D}$ for $n \geq 1$, for every harmonic function $f$ defined on $\mathbb{C}, f \circ g_{n}$ is harmonic on $\mathbb{D}$. Thus for every $k \geq 1$,

$$
\begin{aligned}
\alpha \int_{0}^{1} e^{2 \pi k i \theta} d \nu(\theta) & =\lim _{n \rightarrow \infty} \int_{0}^{1} g_{n}^{k}\left(e^{2 \pi i \theta}\right) d \theta \\
& =\lim _{n \rightarrow \infty} e^{-\alpha k n}=0,
\end{aligned}
$$

the last line using the harmonicity of the function $z \rightarrow z^{k}$. As $\nu$ is a positive measure and for all $k \geq 1$

$$
\int_{0}^{1} e^{2 \pi k i \theta} d \nu(\theta)=0
$$

we conclude that $\nu$ is Lebesgue measure. Hence

$$
\lim _{n \rightarrow \infty} g_{n}\left(\lambda 1_{\mathbb{T} \backslash A}\right)=(1-\alpha) \delta_{0}, \quad \lim _{n \rightarrow \infty} g_{n}\left(\lambda 1_{A}\right)=\alpha \lambda,
$$

with respect to the narrow topology.

We still have to approximate the holomorphic function $g_{n}$ by appropriate polynomials $p_{n}$. First note that it follows by the same argument as above that for every $k \in \mathbb{N}$, the sequence $\left(g_{n}^{k}\right)_{n=1}^{\infty}$ is a sequence in the unit ball of $H^{\infty}(\mathbb{T})$ such that $\left(g_{n}^{k}\left(\lambda 1_{\mathbb{T} \backslash A}\right)\right)_{n=1}^{\infty}$ converges narrowly to $(1-\alpha) \delta_{0}$ and $\left(g_{n}^{k}\left(\lambda 1_{A}\right)\right)_{n=1}^{\infty}$ converges narrowly to $\alpha \lambda$. Let $G_{1}$ be a neighbourhood of $A$ in $\overline{\mathbb{D}}$ which is relatively compact in $G$. As $\left(\left|g_{n}^{k}(z)\right|\right)_{k=1}^{\infty}$ converges to zero uniformly in $z \in \mathbb{D} \backslash G_{1}$ and $n \in \mathbb{N}$ as $k \rightarrow \infty$, we can find $k \in \mathbb{N}$ such that

$$
\left|g_{n}^{k}(z)\right|<\varepsilon / 2 \quad \text { for } n \in \mathbb{N}, \quad z \in \overline{\mathbb{D}} \backslash G_{1} .
$$

Next note that $\lim _{r \rightarrow 1, r<1} g_{n}^{k}(r z)=g_{n}^{k}(z)$ for almost all $z \in \mathbb{T}$. It follows quickly that we may find a sequence $\left(r_{n}\right)_{n=1}^{\infty}$ in $] 0,1[$ tending sufficiently fast to 1 such that the functions $q_{n}(z)=g_{n}^{k}\left(r_{n} z\right)$ verify

(ii) $\left|q_{n}(z)\right|<\varepsilon / 2$ for $z \in \overline{\mathbb{D}} \backslash G_{1}$.

(iii) $\left(q_{n}\left(\lambda 1_{A}\right)\right)_{n=1}^{\infty}$ converges narrowly to $\alpha \lambda$.

(iv) $\left(q_{n}\left(\lambda 1_{\mathbb{T} \backslash A}\right)\right)_{n=1}^{\infty}$ converges narrowly to $(1-\alpha) \delta_{0}$.

Finally find appropriate polynomials $\left(p_{n}\right)_{n=1}^{\infty}$ approximating $\left(q_{n}\right)_{n=1}^{\infty}$ uniformly on $\overline{\mathbb{D}}$ such that the conclusion of the lemma holds true.

We now give an analogue of Proposition II.1 in terms of holomorphic functions instead of analytic martingales. Apparently this result is well known in 
the theory of several complex variables but as we could not find an explicit reference in the literature we include a proof for the sake of completeness, which we formulate in the more general setting of Banach spaces.

Proposition III.3. Let $U$ be a domain in a Banach space $X$ and $f: U \rightarrow \mathbb{R}$ be bounded and uniformly continuous. Define for $x \in U$,

$$
\psi(x)=\inf \int_{0}^{1} f \circ g\left(e^{2 \pi i \theta}\right) d \theta,
$$

where the inf is taken over all $X$-valued polynomials $g$ such that $g(0)=x$ and $g(\overline{\mathbb{D}}) \subseteq U$. Then $\psi$ equals the largest plurisubharmonic function $\hat{f}$ on $U$ dominated by $f$.

Proof. $\psi$ is upper semicontinuous: indeed, let $\left(x_{k}\right)_{k=1}^{\infty}$ in $U$ converge to $x_{0} \in$ $U$ and choose, for $\varepsilon>0$, a polynomial $g: \mathbb{C} \rightarrow X, g(0)=x_{0}, g(\overline{\mathbb{D}}) \subseteq U$ such that

$$
\psi\left(x_{0}\right)>\int_{0}^{1} f \circ g\left(e^{2 \pi i \theta}\right) d \theta-\varepsilon .
$$

There is $k_{0} \in \mathbb{N}$, such that for $k \geq k_{0},\left\{g(\overline{\mathbb{D}})-x_{0}+x_{k}\right\} \subseteq U$ and by Lebesgue's theorem on dominated convergence

$$
\begin{aligned}
\limsup _{k \rightarrow \infty} \psi\left(x_{k}\right) & \leq \limsup _{k \geq \infty, k \rightarrow k_{0}} \int_{0}^{1} f\left(g\left(e^{2 \pi i \theta}\right)-x_{0}+x_{k}\right) d \theta \\
& \leq \int_{0}^{1} f\left(g\left(e^{2 \pi i \theta}\right)\right) d \theta<\psi\left(x_{0}\right)+\varepsilon
\end{aligned}
$$

which shows that $\psi$ is upper semicontinuous.

For every plurisubharmonic function $\phi$ on $U$ with $\phi \leq f$ we have $\phi \leq \psi$ as for every polynomial $g: \mathbb{C} \rightarrow X$ with $g(\overline{\mathbb{D}}) \subseteq U, g(0)=x_{0}$ we have

$$
\phi\left(x_{0}\right) \leq \int_{0}^{1} \phi \circ g\left(e^{2 \pi i \theta}\right) d \theta \leq \int_{0}^{1} f \circ g\left(e^{2 \pi i \theta}\right) d \theta,
$$

whence $\phi\left(x_{0}\right) \leq \psi\left(x_{0}\right)$.

We still have to show the mean value inequality for $\psi$. Let $x_{0} \in U$ and $y_{0} \in X, y_{0} \neq 0$, such that $\left\{x_{0}+\overline{\mathbb{D}} y_{0}\right\} \subseteq U$. We may assume $x_{0}=0$. Fix $\varepsilon>0$ and $M>0$ such that $|f|$ is bounded by $M$ and such that, for $x$, $y \in U,\|x-y\|<\varepsilon / M$ implies $|f(x)-f(y)|<\varepsilon$.

Let $\mathscr{D}$ be a countable subset of the space $\operatorname{Pol}_{0}(\mathbb{C}, X)$ of $X$-valued polynomials $g, g(0)=0$, which is dense with respect to the topology of uniform convergence on $\overline{\mathbb{D}}$.

For $\theta \in \mathbb{T}$ we may find $g_{\theta} \in \mathscr{D}$ such that

$$
\left\{g_{\theta}(\overline{\mathbb{D}})+e^{2 \pi i \theta} y_{0}\right\} \subseteq U,
$$

and

$$
\psi\left(e^{2 \pi i \theta} y_{0}\right)>\int_{0}^{1} f\left(g_{\theta}\left(e^{2 \pi i t}\right)+e^{2 \pi i \theta} y_{0}\right) d t-\varepsilon .
$$

Choose finitely many $\left(\theta_{j}\right)_{j=1}^{m}$ in $\left[0,1\left[\right.\right.$, compact subsets $\left(A_{j}\right)_{j=1}^{m}$ of $\mathbb{T}$ and open subsets $\left(G_{j}\right)_{j=1}^{m}$ of $\overline{\mathbb{D}}$ such that 
(i) $e^{2 \pi i \theta_{j}} \in A_{j} \subseteq G_{j},\left(G_{j}\right)_{j=1}^{m}$ are pairwise disjoint and

$$
\operatorname{diam}\left(G_{j}\right)<\varepsilon / 2 M\left\|y_{0}\right\| \text { for } 1 \leq j \leq m
$$

(ii) $\sum_{j=1}^{m} \lambda\left(A_{j}\right)>1-\varepsilon / M$;

(iii) $\left|\psi\left(e^{2 \pi i \theta} y_{0}\right)-\psi\left(y_{0} e^{2 \pi i \theta_{j}}\right)\right|<\varepsilon$ for $1 \leq j \leq m$ and $e^{2 \pi i \theta} \in A_{j}$;

(iv) $\left\{g_{\theta_{j}}(\overline{\mathbb{D}})+z y_{0}\right\} \subseteq U$ for $1 \leq j \leq m$ and $z \in \bar{G}_{j}$;

which is easily seen to be possible. Define $g_{j}=g_{\theta_{j}}$ for $1 \leq j \leq m$.

Let $L \geq 1$ be a Lipschitz constant for the functions $\left(g_{j}\right)_{j=1}^{m}$ on $\overline{\mathbb{D}}$ and find $0<\delta<\varepsilon / 2 M$ such that, for $K$ denoting the compact set

$$
K=\left\{\overline{\mathbb{D}} y_{0}\right\} \cup\left\{\bigcup_{j=1}^{m} \bigcup_{z \in \bar{G}_{j}}\left\{g_{j}(\overline{\mathbb{D}})+z y_{0}\right\}\right\},
$$

the set

$$
K_{\delta}=\left\{x \in X: \operatorname{dist}_{\|\|}(x, K) \leq \delta\right\},
$$

is contained in $U$. Apply Lemma III.3 to find, for $1 \leq j \leq m$, sequences $\left(p_{j, n}\right)_{n=1}^{\infty}$ of $\mathbb{C}$-valued polynomials mapping $\mathbb{D}$ into $\mathbb{D}$, such that for $1 \leq j \leq$ $m$,

(i) $p_{j, n}(0)=0$, for $n \in \mathbb{N}$;

(ii) $\left|p_{j, n}(z)\right|<\delta / m L$ for $z \in \overline{\mathbb{D}} \backslash G_{j}$ and $n \in \mathbb{N}$;

(iii) $\left(p_{j, n}\left(\lambda 1_{A_{j}}\right)\right)_{n=1}^{\infty}$ converges narrowly to $\lambda\left(A_{j}\right) \lambda$.

Define, for $n \in \mathbb{N}$, the polynomial $h_{n}: \mathbb{C} \rightarrow X$ by

$$
h_{n}(z)=z y_{0}+\sum_{j=1}^{m} g_{j} \circ p_{j, n}(z) \text {. }
$$

Let us check that $h_{n}(\overline{\mathbb{D}}) \subseteq K_{\delta} \subseteq U$. Indeed, if $z \in \overline{\mathbb{D}}, z \notin \bigcup_{j=1}^{m} G_{j}$, then, for $1 \leq j \leq m,\left|p_{j, n}(z)\right|<\delta / m L$, whence

$$
\left\|\sum_{j=1}^{m} g_{j} \circ p_{j, n}(z)\right\|<m L(\delta / m L)=\delta
$$

and therefore $h_{n}(z) \in K_{\delta}$.

If $z \in G_{j_{0}}$, for some $1 \leq j_{0} \leq m$, then

$$
h_{n}(z)=z y_{0}+g_{j_{0}}\left(p_{j_{0}, n}(z)\right)+\sum_{j \neq j_{0}} g_{j} \circ p_{j, n}(z),
$$

whence

$$
\left\|h_{n}(z)-\left(g_{j_{0}}\left(p_{j_{0}, n}(z)\right)-z y_{0}\right)\right\|=\left\|\sum_{j \neq j_{0}} g_{j} \circ p_{j, n}(z)\right\|<\delta,
$$

and therefore again $h_{n}(z) \in K_{\delta}$. 
Finally we may estimate, for $n \in \mathbb{N}$,

$$
\begin{aligned}
\int_{0}^{1} f & \circ h_{n}\left(e^{2 \pi i \theta}\right) d \theta \\
& \leq \sum_{j=1}^{m} \int_{A_{j}} f\left\{e^{2 \pi i \theta} y_{0}+g_{j} \circ p_{j, n}\left(e^{2 \pi i \theta}\right)+\sum_{k \neq j} g_{k} \circ p_{k, n}\left(e^{2 \pi i \theta}\right)\right\} d \theta+\varepsilon \\
& \leq \sum_{j=1}^{m} \int_{A_{j}} f\left\{\left\{g_{j} \circ p_{j, n}\left(e^{2 \pi i \theta}\right)+y_{0} e^{2 \pi i \theta_{j}}\right\}+k_{j}(\theta)\right\} d \theta+\varepsilon,
\end{aligned}
$$

where

$$
k_{j}(\theta)=\sum_{k \neq j} g_{k} \circ p_{k, n}\left(e^{2 \pi i \theta}\right)+\left(e^{2 \pi i \theta}-e^{2 \pi i \theta_{j}}\right) y_{0},
$$

so that, for $\theta \in A_{j},\left\|k_{j}(\theta)\right\|<\varepsilon / 2 M+\delta<\varepsilon / M$.

By the equicontinuity assumption on $f$ we therefore can estimate

$$
\int_{0}^{1} f \circ h_{n}\left(e^{2 \pi i \theta}\right) d \theta \leq \sum_{j=1}^{m} \int_{A_{j}} f\left(g_{j} \circ p_{j, n}\left(e^{2 \pi i \theta}\right)+y_{0} e^{2 \pi i \theta_{j}}\right) d \theta+2 \varepsilon,
$$

passing to $n \rightarrow \infty$ we obtain

$$
\begin{aligned}
\psi(0) & \leq \lim _{n \rightarrow \infty} \sum_{j=1}^{m} \int_{A_{j}} f\left(g_{j} \circ p_{j, n}\left(e^{2 \pi i \theta}\right)+y_{0} e^{2 \pi i \theta_{j}}\right) d \theta+2 \varepsilon \\
& =\sum_{j=1}^{m} \lambda\left(A_{j}\right) \int_{0}^{1} f\left(g_{j}\left(e^{2 \pi i \theta}\right)+y_{0} e^{2 \pi i \theta_{j}}\right) d \theta+2 \varepsilon \\
& \leq \sum_{j=1}^{m} \lambda\left(A_{j}\right) \psi\left(y_{0} e^{2 \pi i \theta_{j}}\right)+3 \varepsilon \\
& \leq \sum_{j=1}^{m} \int_{A_{j}} \psi\left(e^{2 \pi i \theta} y_{0}\right) d \theta+4 \varepsilon \\
& \leq \int_{0}^{1} \psi\left(e^{2 \pi i \theta} y_{0}\right) d \theta+5 \varepsilon
\end{aligned}
$$

the last line using the fact that $\psi \geq-M$. As $\varepsilon>0$ is arbitrary we obtain the mean value inequality for $\psi$ and finish the proof of III.4.

Proof of Theorem (B). Fix $\mu, K, U$ and $x_{0}$ as in Theorem (B). We may assume $x_{0}=0$. We shall first prove Theorem (B) with respect to the class of bounded uniformly continuous functions on $U$. Set

$$
\mathbf{P}=\left\{g(\lambda): g: \mathbb{C} \rightarrow \mathbb{C}^{n} \text { a polynomial, } g(0)=0, g(\overline{\mathbb{D}}) \subseteq U\right\},
$$

which we consider as a subset of $\mathscr{M}^{c}(U)$ the space of Radon measures with compact support in $U$. This space is in duality with the space $C^{\mathrm{ucb}}(U)$ of bounded uniformly continuous functions on $U$ and we equip $\mathscr{M}^{c}(U)$ with the weak topology induced by $C^{\mathrm{ucb}}(U)$.

We now show that the closure of $\mathbf{P}$ is convex. Let $\left(g_{j}\right)_{j=1}^{m}$ be $\mathbb{C}^{n}$-valued polynomials, $g_{j}(0)=0, g_{j}(\overline{\mathbb{D}}) \subseteq U$ for $1 \leq j \leq m$ and $\left(c_{j}\right)_{j=1}^{m}$ positive scalars, $\sum_{j=1}^{m} c_{j}=1$. 
Fix $\varepsilon>0$ and find disjoint compact sets $\left(A_{j}\right)_{j=1}^{m}$ of $\mathbb{T}$ of measure $\lambda\left(A_{j}\right)=$ $(1-\varepsilon) c_{j}$ and disjoint neighbourhoods $\left(G_{j}\right)_{j=1}^{m}$ of $\left(A_{j}\right)_{j=1}^{m}$ in $\overline{\mathbb{D}}$. Fix a norm \|\| on $\mathbb{C}^{n}$, find $\delta>0$ such that $\min _{1 \leq j \leq m} \operatorname{dist}_{\|\|}\left(g_{j}(\overline{\mathbb{D}}), \mathbb{C}^{n} \backslash U\right)>\delta$ and let $L$ be a Lipschitz constant for $\left(g_{j}\right)_{j=1}^{m}$ on $\overline{\mathbb{D}}$.

Now apply Lemma III. 2 to find sequences $\left(p_{j, k}\right)_{k=1}^{\infty}$ of $\mathbb{C}$-valued polynomials such that, for $1 \leq j \leq m$,

(i) $p_{j, k}(0)=0$ for $k \in \mathbb{N}$;

(ii) $\left|p_{j, k}(z)\right|<\delta / L m$ for $z \in \overline{\mathbb{D}} \backslash G_{j}$ and $k \in \mathbb{N}$;

(iii) $\left(p_{j, k}\left(\lambda 1_{A_{j}}\right)\right)_{k=1}^{\infty}$ converges narrowly to $(1-\varepsilon) c_{j} \lambda$;

(iv) $\left(p_{j, k}\left(\lambda 1_{\mathbb{T} \backslash A_{j}}\right)\right)_{k=1}^{\infty}$ converges narrowly to $\left(1-(1-\varepsilon) c_{j}\right) \delta_{0}$. Then

$$
h_{k}=\sum_{j=1}^{m} g_{j} \circ p_{j, k}
$$

is a $\mathbb{C}^{k}$-valued polynomial, $h_{k}(0)=0$ and $h_{k}(\overline{\mathbb{D}})$ is contained in $U$. For every $f \in C^{\mathrm{ucb}}(U)$ we have

$$
\lim _{k \rightarrow \infty}\left\langle f, h_{k}(\lambda)\right\rangle=(1-\varepsilon)\left\langle f, \sum_{j=1}^{m} c_{j} g_{j}(\lambda)\right\rangle,
$$

which readily shows that $\sum_{j=1}^{m} c_{j} g_{j}(\lambda)$ is in the closure of $P$.

Hence similarly as in the proof of Theorem (A) we now are in a position to apply the Hahn-Banach theorem: If $\mu$ were not in the closure of $\mathbf{P}$, then we could find $f \in C^{\mathrm{ucb}}(U)$ and $\alpha<\beta$ such that

$$
\langle f, \mu\rangle=\int_{U} f d \mu \leq \alpha, \quad\langle f, \nu\rangle=\int_{U} f d \nu \geq \beta,
$$

for every $\nu \in A$. The last line can be rewritten as

$$
\int_{\mathbb{T}} f \circ g d \lambda \geq \beta
$$

for every polynomial $g: \mathbb{C} \rightarrow \mathbb{C}^{n}, g(0)=0, g(\overline{\mathbb{D}}) \subseteq U$ whence Proposition III. 3 implies that $\hat{f}(0) \geq \beta$ and we arrive at the desired contradiction:

$$
\alpha \geq\langle f, \mu\rangle \geq\langle\hat{f}, \mu\rangle \geq \hat{f}(0) \geq \beta .
$$

Now for $\varepsilon>0, f_{1}, f_{2}, \ldots, f_{m}$ continuous functions on $U$ bounded by $M>0$, there is $\delta>0$ such that $K+\bar{B}(0, \delta) \subseteq U$, there are $g_{1}, g_{2}, \ldots, g_{m}$ uniformly continuous functions on $U$ bounded by $M$ such that, for $1 \leq i \leq$ $m, f_{i}$ and $g_{i}$ coincide on $K+\bar{B}(0, \delta)$. Note that there is a uniformly continuous function $h$ on $U$ with $0 \leq h \leq 1$, and such that $h$ equals 1 on $U \backslash\{K+\bar{B}(0, \delta)\}$ and equals 0 on $K$. Applying the conclusion of Theorem (B) for $\varepsilon>0, g_{1}, g_{2}, \ldots, g_{m}$ and $h$, we can find a polynomial $p: \mathbb{C} \rightarrow \mathbb{C}^{n}$, $p(0)=0, p(\overline{\mathbb{D}}) \subseteq U$, such that

$$
\begin{gathered}
|\langle h, \mu\rangle-\langle h, p(\lambda)\rangle|<\varepsilon, \\
\left|\left\langle g_{i}, \mu\right\rangle-\left\langle g_{i}, p(\lambda)\right\rangle\right|<\varepsilon \quad \text { for } 1 \leq i \leq m .
\end{gathered}
$$

Note that $\mu$ is supported by $K$ and $h$ vanishes on $K$, so $|\langle h, p(\lambda)\rangle|<\varepsilon$. Note also that $0 \leq h \leq 1$ and $h$ equals 1 on $U \backslash\{K+\bar{B}(0, \delta)\}$, we have then 


$$
\begin{aligned}
p(\lambda)\{U \backslash\{K+\bar{B}(0, \delta)\}\} & \leq \varepsilon, \text { whence } \\
\left|\left\langle f_{i}, \mu\right\rangle-\left\langle f_{i}, p(\lambda)\right\rangle\right| & \leq\left|\left\langle g_{i}, \mu\right\rangle-\left\langle g_{i}, p(\lambda)\right\rangle\right|+2 M p(\lambda)\{U \backslash\{K+\bar{B}(0, \delta)\}\} \\
& \leq \varepsilon+2 M \varepsilon \text { for } 1 \leq i \leq m .
\end{aligned}
$$

This shows that the conclusion of Theorem (B) holds true for $f_{1}, f_{2}, \ldots, f_{m}$.

To end this section we show the compatibility of Definition I. 3 and Definition I.5 above.

Proposition III.4. Let $\mu$ be a probability measure on $\mathbb{C}^{n}$ with compact support. If the inequality

$$
\varphi(0) \leq \int_{\mathbb{C}^{n}} \varphi(x) d \mu(x)
$$

holds true for all Lipschitz plurisubharmonic functions $\varphi$ on $\mathbb{C}^{n}$, then (1) holds true for all plurisubharmonic functions $\varphi$ on $\mathbb{C}^{n}$.

We shall say that a function $f: \mathbb{C}^{n} \rightarrow \mathbb{R}$ is locally Lipschitz if, for every bounded open subset $U$ of $\mathbb{C}^{n},\left.f\right|_{U}$ is a Lipschitz function on $U$. The proof of Proposition III.4 will rely on the following result:

Proposition III.5. Let $\varphi$ be a locally Lipschitz plurisubharmonic function on $\mathbb{C}^{n}$. Then $\varphi$ can be approximated by Lipschitz plurisubharmonic functions uniformly on compact subsets of $\mathbb{C}^{n}$.

Admitting Proposition III.5 it is fairly standard to deduce Proposition III.4:

Proof of Proposition III.4. Fix a plurisubharmonic function $\varphi$ on $\mathbb{C}^{n}$. It follows from the argument in [ $\mathrm{Ra}$, Theorem II.4.12] that there is a decreasing sequence $\left(\varphi_{j}\right)_{j=1}^{\infty}$ of plurisubharmonic $C^{\infty}$-functions on $\mathbb{C}^{n}$ decreasing pointwise to $\varphi$. In particular each $\varphi_{j}$ is locally Lipschitz, hence we may find by Proposition III.5 a sequence $\left(\psi_{j}\right)_{j=1}^{\infty}$ of Lipschitz plurisubharmonic functions on $\mathbb{C}^{n}$ such that $\left|\varphi_{j}-\psi_{j}\right| \leq 1 / j$ on $\operatorname{supp}(\mu) \cup\{0\}$.

Assuming the validity of inequality (1) for every $\psi_{j}$ we obtain

$$
\varphi_{j}(0) \leq \int_{\mathbb{C}^{n}} \varphi_{j}(x) d \mu(x)+2 / j
$$

whence by the Beppo-Levi theorem

$$
\varphi(0) \leq \int_{\mathbb{C}^{n}} \varphi(x) d \mu(x) .
$$

Proof of Proposition III.5. Fix a norm \|\| on $X=\mathbb{C}^{n}$ and a locally Lipschitz plurisubharmonic function $\varphi$ on $X$. We shall approximate $\varphi$ uniformly on $B_{1}=\{x:\|x\| \leq 1\}$ by Lipschitz plurisubharmonic functions $\psi$ on $X$. We may assume that $\left.\varphi\right|_{B_{1}} \geq 0$ and we may find $k_{0} \in \mathbb{N}$ such that $\left.\varphi\right|_{B_{1}}$ obeys a Lipschitz constant less than $k_{0}$.

For $k \geq k_{0}$, let $\varphi_{k}$ be the largest function on $\mathbb{C}^{n}$ satisfying a Lipschitz constant $k$ and coinciding with $\varphi$ on $B_{1}$, and denote by $\hat{\varphi}_{k}$ the plurisubharmonic envelope of $\varphi_{k}$. The sequence $\left(\hat{\varphi}_{k}\right)_{k=k_{0}}^{\infty}$ is an increasing sequence of Lipschitz plurisubharmonic functions on $\mathbb{C}^{n}$ [G-M, Lemma II.1] such that, for every $k \geq k_{0}$,

$$
\left.\hat{\varphi}_{k}\right|_{B_{1}} \leq\left.\varphi\right|_{B_{1}} .
$$


By Dini's theorem it will suffice to prove that $\left(\hat{\varphi}_{k}\right)_{k=k_{0}}^{\infty}$ tends pointwise to $\varphi$ on $B_{1}$.

Let us assume to the contrary that there is $x_{0} \in B_{1}$ and $\alpha<\beta$ such that $\hat{\varphi}_{k}\left(x_{0}\right)<\alpha$ for all $k \geq k_{0}$ while $\varphi\left(x_{0}\right)=\beta$ and let us work towards a contradiction. For $k \geq k_{0}$ we can find, by Proposition III.3, a polynomial $p_{k}: \mathbb{C} \rightarrow X$, $p_{k}(0)=x_{0}$ and such that

$$
\mathbb{E}\left(\varphi_{k} \circ p_{k}\right)=\int_{0}^{1} \varphi_{k}\left(p_{k}\left(e^{2 i \pi \theta}\right)\right) d \theta<\alpha .
$$

Hence for every $\varepsilon>0$

$$
\lim _{k \rightarrow \infty} \mathbb{E}\left\|p_{k} 1_{\left\{\left\|p_{k}\right\|>1+\varepsilon\right\}}\right\|=0 .
$$

Indeed, for every $M \in \mathbb{N}$ there is $k_{1} \geq k_{0}$ such that for $k \geq k_{1}$ and $x \in X$, $\|x\|>1+\varepsilon, \varphi_{k}(x)>M\|x\|$, hence

$$
\lim _{k \rightarrow \infty} \mathbb{E}\left\|p_{k} 1_{\left\{\left\|p_{k}\right\|>1+\varepsilon\right\}}\right\| \leq \lim _{k \rightarrow \infty} M^{-1} \mathbb{E}\left(\varphi_{k} \circ p_{k}\right)<M^{-1} \alpha,
$$

which proves (3). In particular the sequence $\left\{\left\|p_{k}\right\|\right\}_{k=k_{0}}^{\infty}$ is uniformly integrable in $L^{1}(\mathbb{T}, \lambda)$.

Denote by $\left(W_{t}\right)_{t \leq \tau}$ Brownian motion on $\mathbb{C}$ modelled on some probability space $(\Omega, \Sigma, \mathbb{P})$, with $W_{0} \equiv 0$ and stopped at the first time $\tau$ when $\left|W_{\tau}\right|=1$. For $k \geq k_{0}$ denote by $\tau_{k}$ the stopping time

$$
\tau_{k}(\omega)=\tau(\omega) \wedge \inf \left\{t:\left\|p_{k} \circ W_{t}(\omega)\right\| \geq 2\right\},
$$

and let $A_{k}=\left\{\omega \in \Omega: \tau_{k}(\omega)<\tau(\omega)\right\}$. We claim that

$$
\lim _{k \rightarrow \infty} \mathbb{P}\left(A_{k}\right)=0 \text {. }
$$

Indeed, the process $\left\{\left(\left\|p_{k} \circ W_{t}\right\|-3 / 2\right)^{+}\right\}_{t \leq \tau}$ is a submartingale whence

$$
\begin{aligned}
\mathbb{P}\left(A_{k}\right) / 2 & =\mathbb{E}\left(\left(\left\|p_{k} \circ W_{\tau_{k}}\right\|-3 / 2\right)^{+} 1_{A_{k}}\right) \\
& \leq \mathbb{E}\left(\left(\left\|p_{k} \circ W_{\tau}\right\|-3 / 2\right)^{+} 1_{A_{k}}\right) \\
& \leq \mathbb{E}\left(\left(\left\|p_{k} \circ W_{\tau}\right\|-3 / 2\right)^{+}\right) \\
& \leq \mathbb{E}\left(\left\|p_{k} 1_{\left\{\left\|p_{k}\right\|>3 / 2\right\}}\right\|\right),
\end{aligned}
$$

whence (4) follows from (3). Now let $R_{k}$ (resp. $S_{k}$ ) be the $X$-valued random variable $p_{k} \circ W_{\tau}$ (resp. $\left.p_{k} \circ W_{\tau_{k}}\right)$ defined on $(\Omega, \Sigma, \mathbb{P})$. The sequence $\left(R_{k}\right)_{k=k_{0}}^{\infty}$ has the same law as the sequence $\left(p_{k}\right)_{k=k_{0}}^{\infty}$ of random variables on $(\mathbb{T}, \lambda)$, so the sequence $\left(R_{k}\right)_{k=1}^{\infty}$ is uniformly integrable in $L^{1}(\mathbb{P}, X)$. Clearly $\left\|S_{k}(\omega)\right\|$ is bounded by 2 for $k \geq k_{0}$ and $\omega \in \Omega$. We may estimate

$$
\lim _{k \rightarrow \infty} \mathbb{E}\left(\left\|R_{k}-S_{k}\right\|\right) \leq \lim _{k \rightarrow \infty}\left(\mathbb{E}\left(\left\|R_{k} 1_{A_{k}}\right\|\right)+\mathbb{E}\left(\left\|S_{k} 1_{A_{k}}\right\|\right)\right)=0,
$$

whence for every Lipschitz function $f$ on $\mathbb{C}^{n}$

$$
\limsup _{k \rightarrow \infty} \mathbb{E}\left(f \circ R_{k}\right)=\limsup _{k \rightarrow \infty} \mathbb{E}\left(f \circ S_{k}\right) .
$$

The function $\varphi$ is plurisubharmonic whence for $k \geq k_{0}$ the process $\left(\varphi \circ p_{k} \circ W_{t}\right)_{t \leq \tau}$ is a submartingale and therefore

$$
\beta=\varphi\left(x_{0}\right) \leq \mathbb{E}\left(\varphi \circ p_{k} \circ W_{\tau_{k}}\right)=\mathbb{E}\left(\varphi \circ S_{k}\right) .
$$


On the other hand let $m \geq k_{0}$ be big enough such that $\left.\varphi_{m}\right|_{B_{2}} \geq\left.\varphi\right|_{B_{2}}$. Then we may apply (5) and (1) to obtain

$$
\begin{aligned}
\limsup _{k \rightarrow \infty} \mathbb{E}\left(\varphi \circ S_{k}\right) & \leq \limsup _{k \rightarrow \infty} \mathbb{E}\left(\varphi_{m} \circ S_{k}\right) \\
& \leq \limsup _{k \rightarrow \infty} \mathbb{E}\left(\varphi_{m} \circ R_{k}\right) \\
& \leq \limsup _{k \rightarrow \infty} \mathbb{E}\left(\varphi_{k} \circ R_{k}\right) \\
& =\underset{k \rightarrow \infty}{\limsup } \mathbb{E}\left(\varphi_{k} \circ p_{k}\right) \leq \alpha,
\end{aligned}
$$

a contradiction finishing the proof of Proposition III.5.

\section{A VARIATION OF THEOREM (A)}

In order to prove Theorem $(\mathrm{C})$ we shall need a refinement of Theorem (A). The proof involves standard but cumbersome measure theoretical arguments and will be given in this section.

Proposition IV.1. Let $\mu$ be a Jensen measure on a complex Banach space $X$ with barycenter $x_{0}$ and $\varepsilon>0$. Then there is a polynomial $g: \mathbb{C} \rightarrow X$ and $a$ measurable function $f \in L^{1}(\mathbb{T}, X)$ such that

(i) $f(\lambda)=\mu$,

(ii) $g(0)=x_{0}$,

(iii) $\|f-g\|_{1}=\int_{0}^{1}\left\|f(t)-g\left(e^{2 \pi i t}\right)\right\| d \lambda(t)<\varepsilon$.

Before proving Proposition IV.1 we deduce a parametrized version, which will be precisely what we need:

Proposition IV.2. Let $(E, d)$ be a polish space equipped with its Borel $\sigma$-algebra $\Sigma$ and let $\rho$ be a probability measure on $\Sigma$. Let $\left(\mu_{z}\right)_{z \in E}$ be a family of Jensen measures on $X$ with barycenter $\left(x_{z}\right)_{z \in E}$ depending measurably on $z$ in the sense that, for every $\phi \in \operatorname{Lip}(X)$,

$$
z \rightarrow\left\langle\phi, \mu_{z}\right\rangle=\int_{X} \phi(x) d \mu_{z}(x),
$$

is Borel measurable. Then, for $\varepsilon>0$, there is a $\rho$-measurable function

$$
\begin{aligned}
(F, G): X \rightarrow L^{1}(\mathbb{T}, X) \times L^{1}(\mathbb{T}, X), & z \rightarrow\left(f_{z}, g_{z}\right)
\end{aligned}
$$

such that, for every $z \in X, g_{z}$ is the restriction to $\mathbb{T}$ of a polynomial on $\mathbb{C}$ and

(i) $f_{z}(\lambda)=\mu_{z}$,

(ii) $g_{z}(0)=x_{z}$,

(iii) $\left\|f_{z}-g_{z}\right\|<\varepsilon$.

Proof. Define $\mathbf{M}$ to be the subset of $X \times L^{1}(\mathbb{T}, X) \times L^{1}(\mathbb{T}, X)$

$\mathbf{M}=\{(z, f, g): g$ is the restriction to $\mathbb{T}$ of a polynomial on $\mathbb{C}, f(\lambda)=\mu_{z}, g(0)=\mathbb{E}(f)$, and $\left.\|f-g\|<\varepsilon\right\}$. 
The set $\mathbf{M}$ is Borel in $X \times L^{1}(\mathbb{T}, X) \times L^{1}(\mathbb{T}, X)$. Indeed, let $\left(\phi_{n}\right)_{n=4}^{\infty}$ be a sequence of Lipschitz functions on $X$ separating the points of $\mathscr{M}^{1}(X)$ and let

$$
\begin{aligned}
& \mathbf{M}_{1}=\{(z, f, g): g \text { is the restriction to } \mathbb{T} \text { of a polynomial on } \mathbb{C}\}, \\
& \mathbf{M}_{2}=\{(z, f, g):\|f-g\|<\varepsilon\}, \\
& \mathbf{M}_{3}=\{(z, f, g): g(0)=\mathbb{E}(f)\}, \\
& \mathbf{M}_{n}=\left\{(z, f, g):\left\langle\phi_{n}, f(\lambda)\right\rangle=\left\langle\phi_{n}, \mu_{z}\right\rangle\right\} \text { for } n \geq 4 .
\end{aligned}
$$

One can easily check that the above sets are Borel in $X \times L^{1}(\mathbb{T}, X) \times L^{1}(\mathbb{T}, X)$, hence $\mathbf{M}=\bigcap_{n=1}^{\infty} \mathbf{M}_{n}$ is so too.

By Proposition IV.1 the natural projection from $M$ to $X$ is onto hence by a measurable selection theorem (see, e.g., [Co, Theorem 8.5.3] or [H-J, Theorem 9.5]) we can find a $\rho$-measurable selection

$$
(F, G): X \rightarrow L^{1}(\mathbb{T}, X) \times L^{1}(\mathbb{T}, X),
$$

such that, for every $z \in X,(z,(F, G)(z))$ is in $\mathbf{M}$. This means that the function $(F, G)$ satisfies our requirements.

Let us now start to prove Proposition IV.1. Recall first the elementary fact that, if $\mu$ is a positive measure on a polish space $(E, d)$ of mass $\|\mu\|=$ $\mu(E)=\alpha$ and $A$ is a subset of $[0,1]$ of measure $\lambda(A)=\alpha$ then there is a Borel measurable function $f: A \rightarrow E$ such that $f\left(\left.\lambda\right|_{A}\right)=\mu$. The next lemma builds on this observation.

Lemma IV.3. Let $\mu$ be a Radon probability measure on a polish space $(E, d)$, $g:[0,1] \rightarrow E$ Lebesgue measurable and $\varepsilon, \delta>0$. Suppose further that there are disjoint sets $\left(A_{i}\right)_{i=1}^{n}$ of $[0,1]$ and disjoint open sets $\left(B_{i}\right)_{i=1}^{n}$ in $E$ such that, letting $\alpha_{i}=\lambda\left(A_{i}\right)$ and $\beta_{i}=\mu\left(B_{i}\right)$ for $1 \leq i \leq n$

(i) $\sum_{i=1}^{n} \beta_{i}>1-\delta / 2$;

(ii) $\alpha_{i}>\beta_{i}-\delta / 2 n$ for $1 \leq i \leq n$;

(iii) $\operatorname{diam}\left(B_{i}\right)=\sup \left\{d(x, y): x, y \in B_{i}\right\}<\varepsilon$ for $1 \leq i \leq n$;

(iv) $g(t) \in B_{i}$ for $t \in A_{i}$ and $1 \leq i \leq n$.

Then there is a Borel measurable function $f:[0,1] \rightarrow E$ with $f(\lambda)=\mu$ and a measurable subset $C \subseteq[0,1]$ of measure $\lambda(C)>1-\delta$ such that $\|f(t)-g(t)\|<$ $\varepsilon$ on $C$.

Proof. For $1 \leq i \leq n$, let $\gamma_{i}=\min \left(\alpha_{i}, \beta_{i}\right)$, find Borel measurable subsets $C_{i}$ of $A_{i}$ of measure $\lambda\left(C_{i}\right)=\gamma_{i}$ and let $\mu_{i}=\left(\gamma_{i} / \beta_{i}\right) \mu 1_{B_{i}}$, so that the mass of the measures $\mu_{i}$ equals $\left\|\mu_{i}\right\|=\gamma_{i}$. Note that $\sum_{i=1}^{n} \gamma_{i}>1-\delta$. Let $C=\bigcup_{i=1}^{n} C_{i}$, $C_{0}=[0,1] \backslash C$, and $\mu_{0}=\mu-\sum_{i=1}^{n} \mu_{i}$.

For $i=0,1, \ldots, n$ apply the preceding remark to find measurable functions

$$
f_{0}: C_{0} \rightarrow E, \quad f_{i}: C_{i} \rightarrow B_{i} \text { for } 1 \leq i \leq n,
$$

such that

$$
f_{i}\left(\left.\lambda\right|_{C_{i}}\right)=\mu_{i} \text { for } 0 \leq i \leq n .
$$

The function $f=\sum_{i=0}^{n} f_{i} 1_{C_{i}}$ satisfies the requirements.

Proof of Proposition IV.1. As $\mu$ has a first moment we may find $\delta>0$ such that, for $B \subseteq X, \mu(B)<\delta$ we have

$$
\int_{B}\|x\| d \mu(x)<\varepsilon
$$


Next find disjoint compact sets $\left(K_{i}\right)_{i=1}^{n}$ in $X$ of diameter less than $\varepsilon$ such that

$$
\sum_{i=1}^{n} \mu\left(K_{i}\right)>1-\delta / 2,
$$

and open disjoint neighbourhoods $\left(B_{i}\right)_{i=1}^{n}$ of $\left(K_{i}\right)_{i=1}^{n}$ of diameter less than $\varepsilon$ such that $\mu\left(B_{i} \backslash K_{i}\right)<\delta / 4 n$. Now choose Lipschitz functions $\left(\phi_{i}\right)_{i=1}^{n}$ from $X$ to $[0,1]$ such that $\phi_{i}$ equals 1 on $K_{i}$ and vanishes outerside of $B_{i}$.

By Theorem (A) we may find a polynomial $g: \mathbb{C} \rightarrow X$ with $g(0)=x_{0}=$ $\operatorname{bary}(\mu)$ such that

$$
\begin{gathered}
\left|\left\langle\phi_{i}, \mu\right\rangle-\left\langle\phi_{i}, g(\lambda)\right\rangle\right|<\delta / 4 n \text { for } 1 \leq i \leq n, \\
\left|\int_{X}\|x\| d \mu(x)-\int_{X}\|x\| d(g(\lambda))(x)\right|<\varepsilon .
\end{gathered}
$$

For $A_{i}=g^{-1}\left(B_{i}\right)$ let us check that the requirements of Lemma IV.3 are satisfied. The only condition which is not obvious is (ii) which for $1 \leq i \leq n$ follows from

$$
\begin{aligned}
\lambda\left(A_{i}\right) & \geq\left\langle\phi_{i}, g(\lambda)\right\rangle>\left\langle\phi_{i}, \mu\right\rangle-\delta / 4 n \\
& \geq \mu\left(K_{1}\right)-\delta / 4 n \geq \mu\left(B_{i}\right)-\delta / 2 n .
\end{aligned}
$$

Hence we may find $f$ and $C$ as in Lemma IV.3.

Note that it follows from (1) and the relation $\mu=f(\lambda)$ that for any subset $A \subseteq \mathbb{T}$ of measure $\lambda(A)<\delta$ we have

$$
\int_{A}\|f(t)\| d \lambda(t)<\varepsilon .
$$

In order to estimate $\|f-g\|_{1}$ the crucial point is to control the $L^{1}$-mass of $g$ on $C_{0}=\mathbb{T} \backslash C$ :

$$
\begin{aligned}
\int_{C_{0}} & \|g(t)\| d \lambda(t)=\int_{\mathbb{T}}\|g(t)\| d \lambda(t)-\int_{C}\|g(t)\| d \lambda(t) \\
& \leq \int_{\mathbb{T}}\|f(t)\| d \lambda(t)-\int_{C}\|f(t)\| d \lambda(t)+\int_{C}\|f(t)-g(t)\| d \lambda(t)+\varepsilon \\
& <\int_{C_{0}}\|f(t)\| d \lambda(t)+2 \varepsilon<3 \varepsilon,
\end{aligned}
$$

where in the second line we have used (3).

We therefore can estimate

$$
\begin{aligned}
\|f-g\|_{1} & <\int_{C}\|f(t)-g(t)\| d \lambda(t)+\int_{C_{0}}\|f(t)\| d \lambda(t)+\int_{C_{0}}\|g(t)\| d \lambda(t) \\
& <\varepsilon+\varepsilon+3 \varepsilon=5 \varepsilon,
\end{aligned}
$$

thus finishing the proof of Proposition IV.1.

\section{THE PROOF OF THEOREM (C)}

Let $\left(M_{n}\right)_{n=1}^{\infty}$ be an $X$-valued stochastic process defined on a probability space $(\Omega, \Sigma, \mathbb{P})$. Note that there is a standard representation of this process obtained in the following way. Let

$$
\vec{M}: \Omega \rightarrow X^{\mathbb{N}}, \quad \omega \rightarrow\left(M_{n}(\omega)\right)_{n=1}^{\infty},
$$


denote by $\mu$ the image measure $\vec{M}(\mathbb{P})$, by $\pi_{n}: X^{\mathbb{N}} \rightarrow X$ the projection onto the $n$th coordinate and by $\Pi_{n}: X^{\mathbb{N}} \rightarrow X^{n}$ the projection onto the $n$ first coordinates. Equipping $X^{\mathbb{N}}$ with the Borel $\sigma$-algebra $\Sigma$ and letting $\Sigma_{n}$ the $\sigma$-algebra generated by $\Pi_{n}$, the process $\left(\pi_{n}\right)_{i=1}^{\infty}$ on the probability space $\left(X^{\mathbb{N}}, \Sigma, \mu\right)$ is adapted to $\left(\Sigma_{n}\right)_{i=1}^{\infty}$ and has the same law as $\left(M_{n}\right)_{i=1}^{\infty}$.

From now on we suppose that $\left(M_{n}\right)_{i=1}^{n}$ is a martingale and we fix its standard representation, i.e., the measure $\mu$ on $X^{\mathbb{N}}$. For $n \in \mathbb{N}$ we denote by $X_{n}$ the $n$th coordinate of $X^{\mathbb{N}}$, by $\mu_{n}$ the image measure $\mu_{n}=\pi_{n}(\lambda)$ on $X_{n}$, and by $\rho_{n}$ the image measure $\rho_{n}=\Pi_{n}(\lambda)$ on $X^{n} . X^{0}$ will be identified with the one point set $\{0\}$ and $\rho_{0}$ with the unique probability measure on $X^{0}$. The elements $\left(x_{1}, x_{2}, \ldots, x_{n}\right) \in X^{n}$ will occasionally be denoted by $y_{n}$.

By a well-known disintegration theorem (see, e.g., [Schw, Theorem 5.44]) we can, for $n \in \mathbb{N}$, disintegrate $\rho_{n}$ with respect to its marginal $\rho_{n-1}$, i.e., we can find a measurable map

$$
F_{n}: X^{n-1} \rightarrow \mathscr{M}^{1}(X), \quad y_{n-1} \rightarrow \mu_{n, y_{n-1}},
$$

such that

$$
\rho_{n}=\int_{X^{n-1}} \mu_{n, y_{n-1}} d \rho_{n-1}\left(y_{n-1}\right) .
$$

Measurability here means that, for every $f \in \operatorname{Lip}(X)$ the map

$$
\left\langle f, F_{n}()\right\rangle: X^{n-1} \rightarrow \mathbb{R}, \quad y_{n-1} \rightarrow\left\langle f, \mu_{n, y_{n-1}}\right\rangle,
$$

is Borel measurable and formula (1) is a symbolic way of writing that, for every $f \in \operatorname{Lip}\left(X^{n}\right)$ we have

$$
\left\langle f, \rho_{n}\right\rangle=\int_{X^{n-1}} \int_{X_{n}} f\left(y_{n-1}, x_{n}\right) d \mu_{n, y_{n-1}}\left(x_{n}\right) d \rho_{n-1}\left(y_{n-1}\right) .
$$

Proposition V.1. Let $\left(M_{n}\right)_{n=1}^{\infty}$ be a martingale with values in a complex Banach space $X$ and let the probability measure $\mu$ on $X^{\mathbb{N}}$ be its standard representation. Letting $M_{0} \equiv \mathbb{E}\left(M_{1}\right)$ then using the above notation, $\left(M_{n}\right)_{n=1}^{\infty}$ is a plurisubharmonic martingale if and only if $\mu_{1}$ and, for $n \geq 2$ and $\rho_{n-1}$ almost all $y_{n-1} \in X^{n-1}, \mu_{n, y_{n-1}}$ are Jensen measures on $X$.

Proof. First note that, for $n \geq 2$ and for $\rho_{n-1}$ almost all $y_{n-1}=\left(x_{1}, x_{2}, \ldots\right.$, $\left.x_{n-1}\right)$ the barycenter of $\mu_{n, y_{n-1}}$ written symbolically as

$$
\operatorname{bary}\left(\mu_{n, y_{n-1}}\right)=\int_{X_{n}} x_{n} d \mu_{n, y_{n-1}}\left(x_{n}\right),
$$

equals $x_{n-1}$. Indeed let $\left(x_{i}^{*}\right)_{i=1}^{\infty}$ be a sequence in $X^{*}$ separating points of $X$; we have to show that, for $i \in \mathbb{N}$ and for $\rho_{n-1}$ almost all $y_{n-1}=\left(x_{1}, \ldots, x_{n-1}\right)$

$$
\left\langle x_{n-1}, x_{i}^{*}\right\rangle=\int_{X_{n}}\left\langle x_{n}, x_{i}^{*}\right\rangle d \mu_{n, y_{n-1}}\left(x_{n}\right) .
$$

As by assumption $\left(x_{i}^{*} \circ \pi_{n}\right)_{n=1}^{\infty}$ is a martingale on $\left(X^{\mathbb{N}}, \mu\right)$ with respect to the filtration $\left(\Sigma_{n}\right)_{n=1}^{\infty}$ we have for every Borel subset $A \subseteq X^{n-1}$ and every $i \in \mathbb{N}$

$$
\int_{A}\left\langle x_{n-1}, x_{i}^{*}\right\rangle d \rho_{n-1}\left(y_{n-1}\right)=\int_{A} \int_{X_{n}}\left\langle x_{n}, x_{i}^{*}\right\rangle d \mu_{n, y_{n-1}}\left(x_{n}\right) d \rho_{n-1}\left(y_{n-1}\right),
$$

which readily gives $(1)$. 
If now $\mu_{1}$ and, for $n \geq 2$ and $\rho_{n-1}$ almost all $y_{n-1} \in X^{n-1}$ the measure $\mu_{n, y_{n-1}}$ is Jensen on $X$, then it is straightforward to check that $\left(M_{n}\right)_{n=0}^{\infty}$ is a plurisubharmonic martingale.

Conversely, if $\left(M_{n}\right)_{n=1}^{\infty}$ is a plurisubharmonic martingale then it follows immediately that $\mu_{1}$ is a Jensen measure on $X$. Fix $n \geq 2$; we have to show that, for $\rho_{n-1}$ almost all $y_{n-1}=\left(x_{1}, x_{2}, \ldots, x_{n-1}\right) \in \bar{X}^{n-1}$ we have that for all Lipschitz plurisubharmonic functions $\phi$ on $X$

$$
\phi\left(x_{n-1}\right) \leq \int_{X_{n}} \phi\left(x_{n}\right) d \mu_{n, y_{n-1}}\left(x_{n}\right) \text {. }
$$

Let $\left(\phi_{n}\right)_{n=1}^{\infty}$ be a sequence in the set $\mathrm{PSH}_{1}(X)$ of Lipschitz plurisubharmonic functions on $X$ with Lipschitz constant less than 1 that vanish at the origin such that $\left(\phi_{n}\right)_{n=1}^{\infty}$ is dense with respect to the topology of pointwise convergence on $X$. We have to show that the function $\Phi$ on $X^{n-1}$ defined by

$$
\Phi\left(y_{n-1}\right)=\inf _{\phi \in \mathrm{PSH}_{1}(X)}\left\{\int_{X_{n}} \phi\left(x_{n}\right) d \mu_{n, y_{n-1}}\left(x_{n}\right)-\phi\left(x_{n-1}\right)\right\}
$$

is not strictly negative on a set of $\rho_{n-1}$-positive measure. By applying the subsequent Lemma V.2 to the measure $\mu_{n, y_{n-1}}-\delta_{\left\{x_{n-1}\right\}}$ we conclude that

$$
\Phi\left(y_{n-1}\right)=\inf _{i \in \mathbb{N}}\left\{\int_{X_{n}} \phi_{i}\left(x_{n}\right) d \mu_{n, y_{n-1}}\left(x_{n}\right)-\phi_{i}\left(x_{n-1}\right)\right\},
$$

and it will therefore suffice to show that, for every $i \in \mathbb{N}$,

$$
\Phi_{i}\left(y_{n-1}\right)=\int_{X_{n}} \phi_{i}\left(x_{n}\right) d \mu_{n, y_{n-1}}\left(x_{n}\right)-\phi_{i}\left(x_{n-1}\right)
$$

is greater than or equal to zero $\rho_{n-1}$-almost surely. If this were not the case, we could find a Borel set $A \subseteq X^{n-1}$ such that

$$
\int_{A} \Phi_{i}\left(y_{n-1}\right) d \rho_{n-1}\left(y_{n-1}\right)<0
$$

which is contradictory to the assumption that $\left(\phi_{i} \circ \pi_{n}\right)_{n=1}^{\infty}$ is a submartingale on $\left(X^{\mathbb{N}}, \mu\right)$ in view of

$$
\int_{A} \int_{X_{n}} \phi_{i}\left(x_{n}\right) d \mu_{n, y_{n-1}}\left(x_{n}\right) d \rho_{n-1}\left(y_{n-1}\right)<\int_{A} \phi_{i}\left(x_{n-1}\right) d \rho_{n-1}\left(y_{n-1}\right) .
$$

We have used the subsequent lemma whose proof is left to the reader:

Lemma V.2. Let $\mu$ be a finite signed measure on $X$ such that the absolute value $|\mu|$ has a first moment. Let $C$ be a set of functions with Lipschitz constant bounded by 1 and $\bar{C}$ its closure in the topology of pointwise convergence on $X$. Then

$$
\sup _{\phi \in C}\langle\phi, \mu\rangle=\sup _{\phi \in \bar{C}}\langle\phi, \mu\rangle .
$$

Proof of Theorem (C). Let $\left(M_{n}\right)_{n=0}^{\infty}$ be an $X$-valued plurisubharmonic martingale, $M_{0} \equiv x_{0}$. Let $\mu$ be its standard representation on $X^{\mathbb{N}}$ and, using the above notation, $\left(\mu_{n}\right)_{n=1}^{\infty}$ and $\left(\rho_{n}\right)_{n=0}^{\infty}$ the marginals of $\mu$ on $X_{n}$ and $X^{n}$ respectively. We proceed by induction on $n \in \mathbb{N}$.

Let $F_{0} \equiv G_{0} \equiv x_{0}$. For $n=1$ we infer from Proposition V.1 that $\mu_{1}$ is a Jensen measure on $X$ with barycenter $x_{0}$. We may apply Proposition IV.1 
to find $F_{1} \in L^{1}(\mathbb{T}, X)$ and an $X$-valued polynomial $G_{1}$ such that $G_{1}(0)=$ $x_{0}, F_{1}(\lambda)=\mu_{1}$ and

$$
\left\|\left(F_{1}-F_{0}\right)-\left(G_{1}-G_{0}\right)\right\|_{1}<\varepsilon_{1} .
$$

Suppose that we have defined $\left(F_{i}\right)_{i=0}^{n-1}$ and $\left(G_{i}\right)_{i=0}^{n-1}$, such that $\left(G_{i}\right)_{i=0}^{n-1}$ is a Hardy martingale,

$$
\left\|\left(F_{i}-F_{i-1}\right)-\left(G_{i}-G_{i-1}\right)\right\|_{1}<\varepsilon_{1} \text { for } 1 \leq i \leq n-1,
$$

and such that denoting by

$$
\begin{gathered}
\vec{F}_{n-1}: \mathbb{T}^{n-1} \rightarrow X^{n-1}, \\
\vec{F}_{n-1}\left(\theta_{1}, \theta_{2}, \ldots, \theta_{n-1}\right)=\left(F_{1}\left(\theta_{1}\right), F_{2}\left(\theta_{1}, \theta_{2}\right), \ldots, F_{n-1}\left(\theta_{1}, \theta_{2}, \ldots, \theta_{n-1}\right)\right),
\end{gathered}
$$

we have

$$
\vec{F}_{n-1}\left(\lambda^{n-1}\right)=\rho_{n-1},
$$

which means that $\left(F_{i}\right)_{i=0}^{n-1}$ is a representation of $\left(M_{i}\right)_{i=0}^{n-1}$.

Consider the disintegration of $\rho_{n}$ discussed above,

$$
\rho_{n}=\int_{X^{n-1}} \mu_{n, y_{n-1}} d \rho_{n-1}\left(y_{n-1}\right) .
$$

We may suppose by Proposition V.1 that, for every $y_{n-1} \in X^{n-1}, \mu_{n, y_{n-1}}$ is a Jensen measure on $X$ with barycenter $x_{n-1}$, where $y_{n-1}=\left(x_{1}, x_{2}, \ldots, x_{n-1}\right)$. We now can apply Proposition IV.2 for $(E, \rho)=\left(\mathbb{T}^{n-1}, \lambda^{n-1}\right)$ to find $\lambda^{n-1}$ measurable functions

$$
\begin{gathered}
(F, G): \mathbb{T}^{n-1} \rightarrow L^{1}(\mathbb{T}, X) \times L^{1}(\mathbb{T}, X), \\
\left(\theta_{1}, \theta_{2}, \ldots, \theta_{n-1}\right) \rightarrow\left\{f_{\theta_{1}}, \theta_{2}, \ldots, \theta_{n-1}\left(\theta_{n}\right), g_{\theta_{1}, \theta_{2}, \ldots, \theta_{n-1}}\left(\theta_{n}\right)\right\}
\end{gathered}
$$

such that, for every $\left(\theta_{1}, \theta_{2}, \ldots, \theta_{n-1}\right) \in \mathbb{T}^{n-1}, g_{\theta_{1}}, \theta_{2}, \ldots, \theta_{n-1}(\cdot)$ is the restriction to $\mathbb{T}$ of an $X$-valued polynomial defined on $\mathbb{C}$ verifying

such that

$$
g_{\theta_{1}, \theta_{2}, \ldots, \theta_{n-1}}(0)=F_{n-1}\left(\theta_{1}, \theta_{2}, \ldots, \theta_{n-1}\right)
$$

$$
f_{\theta_{1}, \theta_{2}, \ldots, \theta_{n-1}}(\lambda)=\mu_{n, \vec{F}_{n-1}\left(\theta_{1}, \theta_{2}, \ldots, \theta_{n-1}\right)}
$$

and

Now let

and

$$
\left\|f_{\theta_{1}, \theta_{2}, \ldots, \theta_{n-1}}-g_{\theta_{1}, \theta_{2}, \ldots, \theta_{n-1}}\right\|_{1}<\varepsilon_{n} .
$$

$$
\begin{aligned}
G_{n}\left(\theta_{1}, \theta_{2}, \ldots, \theta_{n}\right)= & G_{n-1}\left(\theta_{1}, \theta_{2}, \ldots, \theta_{n-1}\right) \\
& +\left(g_{\theta_{1}}, \theta_{2}, \ldots, \theta_{n-1}\left(\theta_{n}\right)-F_{n-1}\left(\theta_{1}, \theta_{2}, \ldots, \theta_{n-1}\right)\right),
\end{aligned}
$$

then (1) holds true with $(n-1)$ replaced by $n$ in view of (2) and (3). Clearly $\left(G_{i}\right)_{i=0}^{n}$ is a Hardy martingale and we can estimate

$$
\begin{aligned}
\|\left(F_{n}\right. & \left.-F_{n-1}\right)-\left(G_{n}-G_{n-1}\right) \|_{1} \\
& =\int_{\mathbb{T}^{n-1}}\left\|f_{\theta_{1}}, \theta_{2}, \ldots, \theta_{n-1}-g_{\theta_{1}, \theta_{2}, \ldots, \theta_{n-1}}\right\|_{1} d \lambda^{n-1}\left(\theta_{1}, \theta_{2}, \ldots, \theta_{n-1}\right) \\
& <\varepsilon_{n} .
\end{aligned}
$$

This proves Theorem $(\mathrm{C})$. 
With Theorem (C) at our disposition we now can harvest Corollary (D) without any further effort:

Proof of Corollary (D). Let $\left(M_{n}\right)_{n=0}^{\infty}$ be an $L^{1}$-bounded plurisubharmonic martingale (i.e., $\left.\sup _{n}\left\|M_{n}\right\|_{1}<\infty\right)$. Apply Theorem (C) to find a representation $\left(F_{n}\right)_{n=0}^{\infty}$ of $\left(M_{n}\right)_{n=0}^{\infty}$ on $\mathbb{T}^{\mathbb{N}}$ such that $F_{n}$ depends only on the first $n$ coordinates and a Hardy martingale $\left(G_{n}\right)_{n=0}^{\infty}$ such that, for $n \in \mathbb{N}$,

$$
\left\|\left(F_{n}-F_{n-1}\right)-\left(G_{n}-G_{n-1}\right)\right\|_{1}<2^{-n} .
$$

Clearly the process $\left(F_{n}-G_{n}\right)_{n=0}^{\infty}$ converges almost surely by the Borel-Cantelli lemma and $\left(G_{n}\right)_{n=0}^{\infty}$ is $L^{1}$-bounded.

If $X$ has the analytic Radon-Nikodym property then $\left(G_{n}\right)_{n=0}^{\infty}$ converges almost surely [Gar] and therefore $\left(F_{n}\right)_{n=0}^{\infty}$ does so too.

Conversely if $L^{1}$-bounded plurisubharmonic martingales converge almost surely then in particular $L^{1}$-bounded analytic martingales do so, whence by Edgar's theorem [E2] $X$ has the analytic Radon-Nikodym property.

To end this section we give an application of Theorem (C). We say that a complex Banach space $X$ is an AMT (resp. HMT resp. PSH-MT) space if for some $0<p<\infty$ there is a constant $C_{p}$ such that for every $X$-valued analytic (resp. Hardy, resp. plurisubharmonic) martingale $\left(M_{n}\right)_{n=0}^{N}$ and for every predictable process $\left(V_{n}\right)_{n=1}^{N}$ bounded in absolute value by 1 we have the following estimate on the martingale transform:

$$
\left\|\sum_{n=1}^{N} V_{n}\left(M_{n}-M_{n-1}\right)\right\|_{p} \leq C_{p}\left\|M_{n}\right\|_{p} .
$$

We refer to [Gar] for a discussion of these concepts. For example D. J. H. Garling proved that $L^{1}([0,1])$ is an HMP space [Gar, Theorem 10] while G. Pisier [P] has shown that the space of trace class operators on $l^{2}$ fails to be an AMT space. Let us also note that a martingale transform of an analytic (resp. Hardy, resp. plurisubharmonic) martingale is an analytic (resp. Hardy, resp. plurisubharmonic) martingale.

It has been shown by $\mathrm{Xu}[\mathrm{X}]$ that the concepts of AMT and HMT spaces coincide. Xu's result combined with Theorem (C) gives the following result:

Theorem V.3. The concepts of AMT, HMT and PSH-MT spaces coincide.

\section{REFERENCES}

[Bo-Da] J. Bourgain and W. J. Davis, Martingale transforms and complex uniform convexity, Trans. Amer. Math. Soc. 294 (1986), 501-515.

[Bu-Da] A. V. Bukhvalov and A. A. Danilevich, Boundary properties of analytic and harmonic functions with values in Banach space, Mat. Zametki 31 (1982), no. 2, 203-214; English transl. in Math. Notes 31 (1982), 104-110.

[Ch] S. B. Chae, Holomorphy and calculus in normed spaces, Pure Appl. Math., Dekker, New York, 1985.

[Co] D. Cohn, Measure theory, Birkhäuser, Stuttgart, 1980.

[D-G-T] W. J. Davis, D. H. J. Garling, and N. Tomczak-Jaegermann, The complex convexity of complex quasi-normed linear spaces, J. Funct. Anal. 55 (1984), 110-150. 
[Dow] P. N. Dowling, Representation operators and the analytic Radon-Nikodym property in Banach spaces, Proc. Roy. Irish. Acad. 85A (1985), 143-150.

[Du] R. Durret, Brownian motion and martingales in analysis, Wadsworth, Belmont, Calif., 1984.

[E1] G. A. Edgar, Complex martingale convergence, Lecture Notes in Math., vol. 1116, SpringerVerlag, Berlin and New York, 1985, pp. 38-59.

[E2] _ Analytic martingale convergence, J. Funct. Anal. 69 (1986), 268-280.

[E3] _ Extremal integral representations, J. Funct. Anal. 23 (1976), 145-161.

[Gam] T. W. Gamelin, Uniform algebras and Jensen measures, Lecture Notes Series, vol. 32, Cambridge Univ. Press, Cambridge, 1978.

[Gar] D. J. H. Garling, On martingales with values in a complex Banach space, Proc. Cambridge Philos. Soc. 104 (1988), 399-406.

[G-M] N. Ghoussoub and B. Maurey, Plurisubharmonic martingales and barriers in complex quasiBanach spaces, Preprint, 1988.

[H-J] J. Hoffmann-Jorgensen, The theory of analytic spaces, Lecture Notes Ser. in Math., Aarhus Univ., Aarhus, 1970.

[K] S. G. Krantz, Function theory of several complex variables, Pure and Appl. Math. Ser., Wiley, New York, 1982.

[L-T] J. Lindenstrauss and L. Trafriri, Classical Banach spaces. I, Ergebnisse der Math. 92, Springer, 1977.

[P] G. Pisier, Personal communication.

[Ra] M. Range, Holomorphic functions and integrable representations in several complex variables, Springer-Verlag, New York, 1986.

[Schw1] L. Schwartz, Disintegration of measures, Tata Inst. Fund. Res. Stud. Math., Bombay, 1976.

[Schw2] __ Radon measures on arbitrary topological spaces and cylindrical measures, Tata Inst. Fund. Res., Oxford Univ. Press, Oxford, 1973.

[X] Q. H. Xu, Personal communication.

Department of Mathematics, University of Paris ViI, Paris, France

Department of Mathematics, University of Wuhan, Wuhan, China

Institut für Mathematik, Johannes Kepler Universität, A-4040 Linz, Austria 